\title{
CHARACTERIZATION OF RATIONAL TIME-FREQUENCY MULTI-WINDOW GABOR FRAMES AND THEIR DUALS
}

\author{
YAN ZHANG AND YUN-Zhang Li
}

\begin{abstract}
This paper addresses multi-window Gabor frames with rational time-frequency product. Such issue was considered by Zibulski and Zeevi (Appl. Comput. Harmonic Anal. 4 (1997), 188-221) in terms of Zak transform matrix (so-called Zibuski-Zeevi matrix), and by many others. In this paper, we introduce of a new Zak transform matrix. It is different from Zibulski-Zeevi matrix, but more direct and convenient for our purpose. Using such Zak transform matrix we characterize rational time-frequency multi-window Gabor frames (Riesz bases and orthonormal bases), and Gabor duals for a Gabor frame. Some examples are also provided, which show that our Zak transform matrix method is efficient.
\end{abstract}

\section{Introduction}

In the past more than twenty years, the theory of frames has seen great achievements. The notion of frame was first introduced by Duffin and Schaeffer in [18], which is a generalization of basis. And in contrast to a basis, a frame may be "overcomplete". A basis in a Hilbert space allows one to represent each element in a unique way. An overcomplete frame also allows one to represent each element via it, but the representation is not unique. This property plays a significant role in mathematics, signal transmission and modern time-frequency analysis. Gabor frames are a class of important frames among all kinds of frames. Given $x_{0}, w_{0} \in \mathbb{R}$, define the modulation operator $E_{w_{0}}$ and the translation operator $T_{x_{0}}$ on $L^{2}(\mathbb{R})$ respectively by

$$
E_{w_{0}} f(\cdot)=e^{2 \pi i w_{0} \cdot} f(\cdot) \text { and } T_{x_{0}} f(\cdot)=f\left(\cdot-x_{0}\right)
$$

for $f \in L^{2}(\mathbb{R})$. Let $L$ be a positive integer, and let $\mathbf{a}=\left(a_{1}, a_{2}, \ldots, a_{L}\right)$, $\mathbf{b}=\left(b_{1}, b_{2}, \ldots, b_{L}\right)$ with $a_{l}, b_{l}>0$ for $1 \leq l \leq L$. In this paper, we investigate Gabor systems $G(\mathbf{g}, \mathbf{a}, \mathbf{b})$ of the form

$$
G(\mathbf{g}, \mathbf{a}, \mathbf{b})=\left\{E_{m b_{l}} T_{n a_{l}} g_{l}: m, n \in \mathbb{Z}, 1 \leq l \leq L\right\},
$$

Received April 16, 2013; Revised May 16, 2014.

2010 Mathematics Subject Classification. 42C15, 42C40.

Key words and phrases. frame, Gabor frame, multi-window Gabor frame.

Supported by the National Natural Science Foundation of China (Grant No. 11271037) and Beijing Natural Science Foundation (Grant No. 1122008). 
where $\mathbf{g}=\left(g_{1}, g_{2}, \ldots, g_{L}\right)$ with $g_{l} \in L^{2}(\mathbb{R}), 1 \leq l \leq L$.

A Gabor system $G(\mathbf{g}, \mathbf{a}, \mathbf{b})$ is called a (Gabor) frame for $L^{2}(\mathbb{R})$ if there exist positive constants $C_{1}$ and $C_{2}$ such that

$$
C_{1}\|f\|^{2} \leq \sum_{l=1}^{L} \sum_{m, n \in \mathbb{Z}}\left|\left\langle f, E_{m b_{l}} T_{n a_{l}} g_{l}\right\rangle\right|^{2} \leq C_{2}\|f\|^{2}
$$

for $f \in L^{2}(\mathbb{R})$, where $C_{1}$ and $C_{2}$ are called frame bounds; called a tight frame (Parseval frame) for $L^{2}(\mathbb{R})$ if $C_{1}=C_{2}\left(C_{1}=C_{2}=1\right)$ in (1.2); and called a Bessel sequence in $L^{2}(\mathbb{R})$ with Bessel bound $C_{2}$ if the right-hand side inequality in (1.2) holds. A frame $G(\mathbf{g}, \mathbf{a}, \mathbf{b})$ for $L^{2}(\mathbb{R})$ is said to be a Riesz basis for $L^{2}(\mathbb{R})$ if it ceases to be a frame whenever an arbitrary element is removed, and at this time, the frame bounds are called Riesz bounds. The Gabor system (frame) of the form (1.1) is called a single-window Gabor system (frame) if $L=1$, and is called a multi-window Gabor system (frame) if $L>1$. For two Bessel sequences $G(\mathbf{g}, \mathbf{a}, \mathbf{b})$ and $G(\mathbf{h}, \mathbf{a}, \mathbf{b})$ in $L^{2}(\mathbb{R})$ with $\mathbf{g}=\left(g_{1}, g_{2}, \ldots, g_{L}\right)$, $\mathbf{h}=\left(h_{1}, h_{2}, \ldots, h_{L}\right)$, define $\mathcal{S}_{\mathbf{h}, \mathbf{g}}: L^{2}(\mathbb{R}) \rightarrow L^{2}(\mathbb{R})$ by

$$
\mathcal{S}_{\mathbf{h}, \mathbf{g}} f=\sum_{l=1}^{L} \sum_{m, n \in \mathbb{Z}}\left\langle f, E_{m b_{l}} T_{n a_{l}} h_{l}\right\rangle E_{m b_{l}} T_{n a_{l}} g_{l} \text { for } f \in L^{2}(\mathbb{R}) .
$$

Then it is a bounded operator on $L^{2}(\mathbb{R})$. Let $G(\mathbf{g}, \mathbf{a}, \mathbf{b})$ and $G(\mathbf{h}, \mathbf{a}, \mathbf{b})$ be both frames for $L^{2}(\mathbb{R}) . G(\mathbf{h}, \mathbf{a}, \mathbf{b})$ is called a dual of $G(\mathbf{g}, \mathbf{a}, \mathbf{b})$ if $\mathcal{S}_{\mathbf{h}, \mathbf{g}} f=f$ for $f \in L^{2}(\mathbb{R})$. It is easy to check that $G(\mathbf{g}, \mathbf{a}, \mathbf{b})$ is also a dual of $G(\mathbf{h}, \mathbf{a}, \mathbf{b})$ if $G(\mathbf{h}, \mathbf{a}, \mathbf{b})$ is a dual of $G(\mathbf{g}, \mathbf{a}, \mathbf{b})$. So we also say $G(\mathbf{g}, \mathbf{a}, \mathbf{b})$ and $G(\mathbf{h}, \mathbf{a}, \mathbf{b})$ are a pair of dual frames in this case. For two Bessel sequences $G(\mathbf{g}, \mathbf{a}, \mathbf{b})$ and $G(\mathbf{h}, \mathbf{a}, \mathbf{b})$ in $L^{2}(\mathbb{R})$, if $\mathcal{S}_{\mathbf{h}, \mathbf{g}} f=f$ for $f \in L^{2}(\mathbb{R})$, then $G(\mathbf{g}, \mathbf{a}, \mathbf{b})$ and $G(\mathbf{h}, \mathbf{a}, \mathbf{b})$ are a pair of dual frames by the fundamental theory of frames.

Single-window Gabor frames have been studied extensively in the past twenty years and more ([3], [4], [8] and [9]). The theory of multi-window Gabor frame for $L^{2}(\mathbb{R})$ was firstly studied by Zibulski and Zeevi in [21] and Zeevi, Zibulski and Porat in [20]. By introduction of a Zak transform, they developed a matrix (so-called Zibulski-Zeevi matrix) algebraic tool for multi-window Gabor frames, and applied it to image processing and computer vision. Those results indicate that the multi-window approach is efficient and superior to the single-window one. Then many researchers studied the theory of multi-window Gabor frames and related applications ([10]-[13], [19] and [20]). Interestingly, these works mainly concern the case that all window functions have the same time and frequency shifts, i.e.,

$$
a_{1}=a_{2}=\cdots=a_{L} \text { and } b_{1}=b_{2}=\cdots=b_{L} .
$$

In practical problems, time and frequency shifts may vary with the windows. Zibulski-Zeevi matrix method was also used in the study of subspace singlewindow Gabor frames ([1], [5] and [6]). In [7] and [16], a different Zak transform and Zak transform matrix from those in [20] and [21] were introduced and used 
effectively to study Gabor systems on periodic subsets of the real line, while Zibulski-Zeevi matrix method does not work well for such Gabor systems. A variation of the method in [7] and [16] was applied to Gabor systems on discrete periodic sets ([14] and [15]). It was also pointed out in [20] that Zibulski-Zeevi matrix method is not very efficient for Gabor frames $G(\mathbf{g}, \mathbf{a}, \mathbf{b})$ of the form (1.1) when (1.3) does not hold. The aim of this paper is to characterize Gabor frames of the form (1.1) and their Gabor duals under the following assumptions:

Assumption 1. $L$ is a positive integer greater than 1 ;

Assumption 2. $b_{1}=b_{2}=\cdots=b_{L}=\beta$, and $a_{l} \beta=\frac{p_{l}}{q_{l}}$ with $p_{l}$ and $q_{l}$ being relatively prime positive integers for $1 \leq l \leq L$.

Our main novelty is to introduce a new Zak transform matrix method, and use it to characterize Gabor frames of the form (1.1) and their Gabor duals. Examples in Section 4 below show that such Zak transform matrix method is more efficient than Zibulski-Zeevi matrix method when treating many rational time-frequency multi-window Gabor frames of the form (1.1). In addition, when $a_{1}=a_{2}=\cdots=a_{L}=\alpha$ and $b_{1}=b_{2}=\cdots=b_{L}=\beta$, Remark 1.2 below shows that, using our Zak transform matrix method than Zibulski-Zeevi matrix method, we can more easily obtain a frame (a Riesz basis, an orthonormal basis) $G(\mathbf{g}, \mathbf{a}, \mathbf{b})$ and its duals $G(\mathbf{h}, \mathbf{a}, \mathbf{b})$ by designing Zak transform matrices $\Phi_{\mathbf{g}}$ and $\Phi_{\mathbf{h}}$.

We denote by $p$ the least common multiple of $p_{l}$ and by $q$ the greatest common divisor of $q_{l}, 1 \leq l \leq L$. Then $p$ and $q$ are relatively prime, and there exist unique $\lambda_{1}, \lambda_{2}, \ldots, \lambda_{L} \in \mathbb{N}$ (N denotes the set of positive integers) such that $\frac{p}{q}=\frac{\lambda_{l} p_{l}}{q_{l}}$ for $1 \leq l \leq L$. So $\lambda_{l} a_{l}=\frac{p}{\beta q}$ for $1 \leq l \leq L$ by Assumption 2. We write $\alpha=\frac{p}{\beta q}$. The following remark tells us Assumptions 1 and 2 are reasonable to some extent. We also remark that the restriction of "rational time-frequency" here is for using "finite-order" Zak transform matrix-valued functions.

Remark 1.1. Assumptions 1 and 2 are relatively general. Suppose $b_{1}, b_{2}, \ldots$, and $b_{L}$ are commensurable, i.e., there exist $\beta>0$ and positive integers $\beta_{1}, \beta_{2}, \ldots$, and $\beta_{L}$ such that $\beta=\beta_{l} b_{l}$ for $1 \leq l \leq L$. Observe that $\mathbb{Z}=\beta_{l} \mathbb{Z}+\left\{0,1, \ldots, \beta_{l}-\right.$ 1) for each $1 \leq l \leq L$. $G(\mathbf{g}, \mathbf{a}, \mathbf{b})$ of the form (1.1) is a frame (a Riesz basis, an orthonomal basis) for $L^{2}(\mathbb{R})$ if and only if

$$
\left\{e^{2 \pi i m \beta} \cdot g_{l}^{\left(\tau_{l}\right)}\left(\cdot-n a_{l}\right): 1 \leq l \leq L, 0 \leq \tau_{l} \leq \beta_{l}-1, m, n \in \mathbb{Z}\right\}
$$

is a frame (a Riesz basis, an orthonomal basis) for $L^{2}(\mathbb{R})$, where $g_{l}^{\left(\tau_{l}\right)}(\cdot)=$ $e^{2 \pi i \tau_{l} b_{l} \cdot} g_{l}(\cdot)$. It is obvious that (1.4) satisfies Assumptions 1 and 2 with another " $L$ ". In particular, $b_{1}, b_{2}, \ldots, b_{L}$ are commensurable if they are all rational numbers or rational multiples of some fixed irrational number. In what follows, unless otherwise specified, we always work under Assumptions 1 and 2 .

For statement of our main results and later use, we introduce some notations and notions. Given $M \in \mathbb{N}$, we denote by $\mathbb{N}_{M}$ the set $\{0,1, \ldots, M-1\}$, by $I_{M}$ 
the $M \times M$ identity matrix (by $I$ the identity matrix if we need not specify its size), by $x_{k}$ the $k$-th component of $x$ for $x \in \mathbb{C}^{M}$, and by $\mathbf{e}_{\mathbf{k}}$ the vector in $\mathbb{C}^{M}$ with the $k$-th component being 1 and the others being zero. For an arbitrary complex matrix $A$, we always denote by $A^{*}$ its conjugate transpose. Given a measurable set $S$ in $\mathbb{R}$, a collection $\left\{S_{k}: k \in \mathbb{Z}\right\}$ of measurable sets in $\mathbb{R}$ is called a partition of $S$ if

$$
\bigcup_{k \in \mathbb{Z}} S_{k}=S \text { and } S_{k} \cap S_{k^{\prime}}=\emptyset \text { for } k \neq k^{\prime} \text { in } \mathbb{Z}
$$

up to a set of measure zero. For $\lambda>0$ and measurable sets $S, S^{\prime} \subset \mathbb{R}$, we say $S$ is $\lambda \mathbb{Z}$-congruent to $S^{\prime}$ if there exists a partition $\left\{S_{k}: k \in \mathbb{Z}\right\}$ of $S$ such that $\left\{S_{k}+\lambda k: k \in \mathbb{Z}\right\}$ is a partition of $S^{\prime}$. In particular, only finitely many $S_{k}$ among $S_{k}, k \in \mathbb{Z}$, are nonempty if both $S$ and $S^{\prime}$ are bounded in addition. Obviously, $S^{\prime}$ is also $\lambda \mathbb{Z}$-congruent to $S$ if $S$ is $\lambda \mathbb{Z}$-congruent to $S^{\prime}$. So we usually say $S$ and $S^{\prime}$ are $\lambda \mathbb{Z}$-congruent in this case. Let us recall the definition of Zak transform in [7]. The Zak transform $\mathcal{Z}_{\alpha q}: L^{2}(\mathbb{R}) \rightarrow L_{l o c}^{2}\left(\mathbb{R}^{2}\right)$ is defined by

$$
\mathcal{Z}_{\alpha q} f(t, v)=\sum_{k \in \mathbb{Z}} f(t+k \alpha q) e^{2 \pi i k v}
$$

for $f \in L^{2}(\mathbb{R})$. It is easy to check that $\mathcal{Z}_{\alpha q}$ has the quasi-periodicity:

(1.5) $\mathcal{Z}_{\alpha q} f(t+m \alpha q, v+n)=e^{-2 \pi i m v} \mathcal{Z}_{\alpha q} f(t, v)$ for $f \in L^{2}(\mathbb{R})$ and $m, n \in \mathbb{Z}$.

Define $\mathbf{Z}_{\alpha q}: L^{2}(\mathbb{R}) \rightarrow L_{\text {loc }}^{2}\left(\mathbb{R}^{2}, \mathbb{C}^{p}\right)$ by

$$
\mathbf{Z}_{\alpha q} f(t, v)=\left(\begin{array}{c}
\mathcal{Z}_{\alpha q} f(t, v) \\
\mathcal{Z}_{\alpha q} f\left(t+\frac{1}{\beta}, v\right) \\
\vdots \\
\mathcal{Z}_{\alpha q} f\left(t+\frac{p-1}{\beta}, v\right)
\end{array}\right)
$$

for $f \in L^{2}(\mathbb{R})$. Then we have:

Lemma 1.1 ([7, Lemma 2.1]). $\mathcal{Z}_{\alpha q}$ is a unitary operator from $L^{2}(\mathbb{R})$ onto $L^{2}([0, \alpha q) \times[0,1))$, and $\mathbf{Z}_{\alpha q}$ is a unitary operator from $L^{2}(\mathbb{R})$ onto $L^{2}\left(\left[0, \frac{1}{\beta}\right) \times\right.$ $\left.[0,1), \mathbb{C}^{p}\right)$.

Definition 1.1. For $\mathbf{g}=\left(g_{1}, g_{2}, \ldots, g_{L}\right)$ with $g_{l} \in L^{2}(\mathbb{R})$, we associate it with the matrix-valued function $\Phi_{\mathrm{g}}: \mathbb{R}^{2} \rightarrow \mathcal{M}_{Q, p}$ defined by

$$
\Phi_{\mathbf{g}}(t, v)=\left(\begin{array}{c}
G_{1}(t, v) \\
G_{2}(t, v) \\
\vdots \\
G_{L}(t, v)
\end{array}\right)
$$


for a.e. $(t, v) \in \mathbb{R}^{2}$, where $Q=q \sum_{l=1}^{L} \lambda_{l}$,

$$
G_{l}(t, v)=\left(\begin{array}{c}
\mathcal{G}_{l}(t, v) \\
\mathcal{G}_{l}\left(t-a_{l}, v\right) \\
\vdots \\
\mathcal{G}_{l}\left(t-\left(\lambda_{l}-1\right) a_{l}, v\right)
\end{array}\right)
$$

and

$$
\mathcal{G}_{l}(t, v)_{r, k}=\mathcal{Z}_{\alpha q} g_{l}\left(t+\frac{k}{\beta}-r \alpha, v\right), r \in \mathbb{N}_{q}, k \in \mathbb{N}_{p}
$$

for $1 \leq l \leq L$.

Similarly, for $\mathbf{h}=\left(h_{1}, h_{2}, \ldots, h_{L}\right)$ with $h_{l} \in L^{2}(\mathbb{R})$, we associate it with $\Phi_{\mathbf{h}}(t, v)$ as in (1.7). The main results of this paper are stated as follows.

Theorem 1.1. $G(\mathbf{g}, \mathbf{a}, \mathbf{b})$ is a frame for $L^{2}(\mathbb{R})$ with frame bounds $A, B$ if and only if

$$
\beta A I \leq \Phi_{\mathbf{g}}^{*}(t, v) \Phi_{\mathbf{g}}(t, v) \leq \beta B I \text { for a.e. }(t, v) \in\left[0, \frac{1}{\beta q}\right) \times[0,1) .
$$

Theorem 1.2. $G(\mathbf{g}, \mathbf{a}, \mathbf{b})$ is a Riesz basis (an orthonormal basis) for $L^{2}(\mathbb{R})$ with Riesz bounds $A, B$ if and only if $p=Q$ and

$$
\beta A I \leq \Phi_{\mathbf{g}}^{*}(t, v) \Phi_{\mathbf{g}}(t, v) \leq \beta B I\left(\Phi_{\mathbf{g}}^{*}(t, v) \Phi_{\mathbf{g}}(t, v)=\beta I\right)
$$

for a.e. $(t, v) \in\left[0, \frac{1}{\beta q}\right) \times[0,1)$.

Theorem 1.3. Let $G(\mathbf{g}, \mathbf{a}, \mathbf{b})$ be a frame for $L^{2}(\mathbb{R})$, and $G(\mathbf{h}, \mathbf{a}, \mathbf{b})$ a Bessel sequence in $L^{2}(\mathbb{R})$. Then $G(\mathbf{h}, \mathbf{a}, \mathbf{b})$ is a dual of $G(\mathbf{g}, \mathbf{a}, \mathbf{b})$ if and only if $\Phi_{\mathbf{g}}^{*}(t, v) \Phi_{\mathbf{h}}(t, v)=\beta I$ for a.e. $(t, v) \in\left[0, \frac{1}{\beta q}\right) \times[0,1)$.

Remark 1.2. Let us make an additional assumption that

$$
a_{1}=a_{2}=\cdots=a_{L}=\alpha \text { and } b_{1}=b_{2}=\cdots=b_{L}=\beta
$$

in Theorems 1.1-1.3. A simple computation shows that

$$
\left(\left[0, \frac{1}{\beta q}\right)+\frac{k}{\beta}-r \alpha\right) \bigcap\left(\left[0, \frac{1}{\beta q}\right)+\frac{k^{\prime}}{\beta}-r^{\prime} \alpha\right)=\emptyset
$$

for $(k, r) \neq\left(k^{\prime}, r^{\prime}\right)$ in $\mathbb{N}_{p} \times \mathbb{N}_{q}$, and the set $\bigcup_{k \in \mathbb{N}_{p}} \bigcup_{r \in \mathbb{N}_{q}}\left(\left[0, \frac{1}{\beta q}\right)+\frac{k}{\beta}-r \alpha\right)$ is $\alpha q \mathbb{Z}$-congruent to [0, $\alpha q)$. It follows that $\mathbf{g}$ and $\mathbf{h}$ are uniquely determined by the values of $\Phi_{\mathbf{g}}$ and $\Phi_{\mathbf{h}}$ on $\left[0, \frac{1}{\beta q}\right) \times[0,1)$ by Lemma 1.1 and the quasiperiodicity of $\mathcal{Z}_{\alpha q}$, and that the values taken by entries of the different position of $\Phi_{\mathbf{g}}\left(\Phi_{\mathbf{h}}\right)$ are unrelated when restricted on $\left[0, \frac{1}{\beta q}\right) \times[0,1)$. This gives us much freedom to obtain $\mathbf{g}$ and $\mathbf{h}$ by designing $\Phi_{\mathbf{g}}$ and $\Phi_{\mathbf{h}}$. Therefore, Theorems 1.11.3 provide us with a new idea for constructing Gabor frames and their duals in addition to Zibulski-Zeevi matrix method. 
The rest of this paper is organized as follows. In Section 2, we give some auxiliary results for later use. Based on Section 2, Section 3 is devoted to proving Theorems 1.1-1.3. Section 4 focuses on some examples of Theorems 1.1-1.3. These general examples show that our matrix algebraic method is efficient for dealing with many rational time-frequency multi-window Gabor frames.

\section{Some auxiliary results}

This section is an auxiliary one to following sections. We start with some lemmas and theorems.

By the quasi-periodicity of $\mathcal{Z}_{\alpha q}$, we have:

Lemma 2.1. Let $f \in L^{2}(\mathbb{R})$ and $x_{0} \in \mathbb{R}$. Then

$\mathcal{Z}_{\alpha q}\left(E_{m \beta} T_{n \alpha q+x_{0}} f\right)(t, v)=e^{2 \pi i m \beta t} e^{2 \pi i n v} \mathcal{Z}_{\alpha q} f\left(t-x_{0}, v\right)$ for a.e. $(t, v) \in \mathbb{R}^{2}$.

Lemma 2.2. Let $\mathbf{g}=\left(g_{1}, g_{2}, \ldots, g_{L}\right)$ with $g_{l} \in L^{2}(\mathbb{R})$. Then

$$
\begin{aligned}
& \left\langle f, E_{m \beta} T_{n \alpha q+r \alpha+\tau_{l} a_{l}} g_{l}\right\rangle \\
= & \int_{0}^{\frac{1}{\beta}} \int_{0}^{1}\left(\overline{\mathcal{G}_{l}\left(t-\tau_{l} a_{l}, v\right)} \mathbf{Z}_{\alpha q} f(t, v)\right)_{r} e^{-2 \pi i m \beta t} e^{-2 \pi i n v} d t d v
\end{aligned}
$$

for $f \in L^{2}(\mathbb{R}), 1 \leq l \leq L$ and $\left(m, n, r, \tau_{l}\right) \in \mathbb{Z} \times \mathbb{Z} \times \mathbb{N}_{q} \times \mathbb{N}_{\lambda_{l}}$.

Proof. By Lemmas 1.1 and 2.1,

$$
\begin{aligned}
& \left\langle f, E_{m \beta} T_{n \alpha q+r \alpha+\tau_{l} a_{l}} g_{l}\right\rangle \\
= & \int_{0}^{\frac{1}{\beta}} \int_{0}^{1} \sum_{k=0}^{p-1} \overline{\mathcal{Z}_{\alpha q} g_{l}\left(t-\tau_{l} a_{l}-r \alpha+\frac{k}{\beta}, v\right)} \mathcal{Z}_{\alpha q} f\left(t+\frac{k}{\beta}, v\right) e^{-2 \pi i m \beta t} e^{-2 \pi i n v} d t d v \\
= & \int_{0}^{\frac{1}{\beta}} \int_{0}^{1}\left(\overline{\mathcal{G}_{l}\left(t-\tau_{l} a_{l}, v\right)} \mathbf{Z}_{\alpha q} f(t, v)\right)_{r} e^{-2 \pi i m \beta t} e^{-2 \pi i n v} d t d v
\end{aligned}
$$

for each $r \in \mathbb{N}_{q}, \tau_{l} \in \mathbb{N}_{\lambda_{l}}$ and $1 \leq l \leq L$. This gives the lemma.

Lemma 2.3. For $\mathbf{g}=\left(g_{1}, g_{2}, \ldots, g_{L}\right)$ with $g_{l} \in L^{2}(\mathbb{R})$, we have

$$
\Phi_{\mathbf{g}}\left(t+\frac{j}{\beta q}, v\right)=e^{-2 \pi i m_{j} v} \mathcal{C}_{j} \Phi_{\mathbf{g}}(t, v) \mathcal{D}_{j}
$$

for a.e. $(t, v) \in \mathbb{R}^{2}$ and $j \in \mathbb{Z}$ with $j=k_{j} q+\left(m_{j} q-r_{j}\right) p,\left(k_{j}, r_{j}, m_{j}\right) \in$ $\mathbb{N}_{p} \times \mathbb{N}_{q} \times \mathbb{Z}$, where $\mathcal{D}_{j}=\left(\begin{array}{cc}0 & e^{-2 \pi i v} I_{k_{j}} \\ I_{p-k_{j}} & 0\end{array}\right), \mathcal{C}_{j}=\operatorname{diag}\left(\mathcal{C}_{1, j}, \mathcal{C}_{2, j}, \ldots, \mathcal{C}_{L, j}\right), \mathcal{C}_{l, j}$ denotes the block matrix (with $\lambda_{l}$ blocks) of the form $\operatorname{diag}\left(C_{j}, C_{j}, \ldots, C_{j}\right)$ with $C_{j}=\left(\begin{array}{cc}0 & I_{q-r_{j}} \\ e^{2 \pi i v} I_{r_{j}} & 0\end{array}\right)$ for $1 \leq l \leq L$. 
Proof. By the same procedure as in [7, Lemma 2.6] and [15, Lemma 5], we can prove that

$$
\mathcal{G}_{l}\left(t+\frac{j}{\beta q}, v\right)=e^{-2 \pi i m_{j} v} C_{j} \mathcal{G}_{l}(t, v) \mathcal{D}_{j} \text { for }(t, v) \in \mathbb{R}^{2} .
$$

This gives the lemma.

Since every $j \in \mathbb{Z}$ has a unique decomposition $j=k_{j} q+\left(m_{j} q-r_{j}\right) p$ with $\left(k_{j}, r_{j}, m_{j}\right) \in \mathbb{N}_{p} \times \mathbb{N}_{q} \times \mathbb{Z}$, as an immediate consequence of Lemma 2.3 we have:

Lemma 2.4. Let $\mathbf{g}=\left(g_{1}, g_{2}, \ldots, g_{L}\right)$ with $g_{l} \in L^{2}(\mathbb{R})$, and let $C$ be a positive constant. Then

$$
\Phi_{\mathbf{g}}^{*}(t, v) \Phi_{\mathbf{g}}(t, v) \geq C I(\leq C I)
$$

holds for a.e. $(t, v) \in \mathbb{R}^{2}$ if and only if it holds for a.e. $(t, v) \in\left[0, \frac{1}{\beta q}\right) \times[0,1)$.

Definition 2.1. Given $c=\left(c_{1}, c_{2}, \ldots, c_{L}\right)$ with $c_{l} \in l^{2}\left(\mathbb{Z}^{2}\right)$, for each $1 \leq l \leq$ $L$, we associate it with a vector-valued function $c(t, v)$ defined by

$$
c(t, v)=\left(\begin{array}{c}
c_{1}(t, v) \\
c_{2}(t, v) \\
\vdots \\
c_{L}(t, v)
\end{array}\right) \quad \text { for a.e. }(t, v) \in \mathbb{R}^{2}
$$

where

and

$$
c_{l}(t, v)=\left(\begin{array}{c}
c_{l}^{(0)}(t, v) \\
c_{l}^{(1)}(t, v) \\
\vdots \\
c_{l}^{\left(\lambda_{l}-1\right)}(t, v)
\end{array}\right)
$$

$$
c_{l}^{\left(\tau_{l}\right)}(t, v)=\left(\begin{array}{c}
\sum_{m, n \in \mathbb{Z}} c_{l, m, n q \lambda_{l}+\tau_{l}} e^{2 \pi i m \beta t} e^{2 \pi i n v} \\
\sum_{m, n \in \mathbb{Z}} c_{l, m,(n q+1) \lambda_{l}+\tau_{l}} e^{2 \pi i m \beta t} e^{2 \pi i n v} \\
\vdots \\
\sum_{m, n \in \mathbb{Z}} c_{l, m,(n q+q-1) \lambda_{l}+\tau_{l}} e^{2 \pi i m \beta t} e^{2 \pi i n v}
\end{array}\right)
$$

for $1 \leq l \leq L$ and $\tau_{l} \in \mathbb{N}_{\lambda_{l}}$.

Similarly, for $d=\left(d_{1}, d_{2}, \ldots, d_{L}\right)$ with $d_{l} \in l^{2}\left(\mathbb{Z}^{2}\right)$, we associate it with $d(t, v)$ as in $(2.3)$.

Lemma 2.5. Given $\mathbf{g}=\left(g_{1}, g_{2}, \ldots, g_{L}\right)$ with $g_{l} \in L^{2}(\mathbb{R})$, let $G(\mathbf{g}, \mathbf{a}, \mathbf{b})$ be a Bessel sequence in $L^{2}(\mathbb{R})$. Then, for $f \in L^{2}(\mathbb{R})$ and $c=\left(c_{1}, c_{2}, \ldots, c_{L}\right)$ with $c_{l} \in l^{2}\left(\mathbb{Z}^{2}\right), f=\sum_{l=1}^{L} \sum_{m, n \in \mathbb{Z}} c_{l, m, n} E_{m \beta} T_{n a_{l}} g_{l}$ if and only if $\mathbf{Z}_{\alpha q} f(t, v)=$ $\overline{\Phi_{\mathrm{g}}^{*}(t, v)} c(t, v)$ for a.e. $(t, v) \in\left[0, \frac{1}{\beta}\right) \times[0,1)$. 
Proof. Since $\mathbb{Z}=\left(q \mathbb{Z}+\mathbb{N}_{q}\right) \lambda_{l}+\mathbb{N}_{\lambda_{l}}$ and $\lambda_{l} a_{l}=\alpha$ for $1 \leq l \leq L, f=$ $\sum_{l=1}^{L} \sum_{m, n \in \mathbb{Z}} c_{l, m, n} E_{m \beta} T_{n a_{l}} g_{l}$ can be written as

$$
f=\sum_{l=1}^{L} \sum_{r=0}^{q-1} \sum_{\tau_{l}=0}^{\lambda_{l}-1} \sum_{m, n \in \mathbb{Z}} c_{l, m,(n q+r) \lambda_{l}+\tau_{l}} E_{m \beta} T_{n \alpha q+r \alpha+\tau_{l} \alpha_{l}} g_{l},
$$

which is equivalent to

$$
\text { 4) } \begin{aligned}
& \mathbf{Z}_{\alpha q} f(t, v) \\
= & \sum_{l=1}^{L} \sum_{\tau_{l}=0}^{\lambda_{l}-1} \sum_{r=0}^{q-1} \sum_{m, n \in \mathbb{Z}} c_{l, m,(n q+r) \lambda_{l}+\tau_{l}} \mathbf{Z}_{\alpha q}\left(E_{m \beta} T_{\alpha q+r \alpha+\tau_{l} a_{l}} g_{l}\right)(t, v) \\
= & \sum_{l=1}^{L} \sum_{\tau_{l}=0}^{\lambda_{l}-1} \sum_{r=0}^{q-1}\left(\sum_{m, n \in \mathbb{Z}} c_{l, m,(n q+r) \lambda_{l}+\tau_{l}} e^{2 \pi i m \beta t} e^{2 \pi i n v}\right) \mathbf{Z}_{\alpha q} g_{l}\left(t-r \alpha-\tau_{l} a_{l}, v\right)
\end{aligned}
$$

for $(t, v) \in\left[0, \frac{1}{\beta}\right) \times[0,1)$ by Lemmas 1.1 and 2.1. By the definition of $\Phi_{\mathbf{g}}(t, v)$, (2.4) can be rewritten as

$$
\mathbf{Z}_{\alpha q} f(t, v)=\overline{\Phi_{\mathbf{g}}^{*}(t, v)} c(t, v) \text { for }(t, v) \in\left[0, \frac{1}{\beta}\right) \times[0,1) .
$$

The proof is completed.

Definition 2.2. Let $G(\mathbf{g}, \mathbf{a}, \mathbf{b})$ be a Bessel sequence in $L^{2}(\mathbb{R})$. We say that $G(\mathbf{g}, \mathbf{a}, \mathbf{b})$ has Riesz property if, for $c=\left(c_{1}, c_{2}, \ldots, c_{L}\right)$ with $c_{l} \in l^{2}\left(\mathbb{Z}^{2}\right)$, whenever

$$
\sum_{l=1}^{L} \sum_{m, n \in \mathbb{Z}} c_{l, m, n} E_{m \beta} T_{n a_{l}} g_{l}=0,
$$

we must have $c=0$, i.e., $c_{l, m, n}=0$ for $1 \leq l \leq L$ and $m, n \in \mathbb{Z}$.

By an easy application of the spectral theorem of self-adjoint matrices, we have the following lemma (see also [2, p. 978]):

Lemma 2.6. Given a measurable set $E$ in $\mathbb{R}^{2}$ with $|E|>0$, let $\mathcal{A}: E \rightarrow$ $\mathcal{M}_{s, t}$ be a matrix-valued measurable function. Define by $\mathcal{P}(t, v)$ the orthogonal projection of $\mathbb{C}^{t}$ onto $\operatorname{ker}(\mathcal{A}(t, v))$. Then

$$
\mathcal{P}(t, v)=\lim _{n \rightarrow \infty} \exp \left(-n \mathcal{A}^{*}(t, v) \mathcal{A}(t, v)\right)
$$

for $(t, v) \in E$, and thus $\mathcal{P}(t, v)$ is measurable.

Theorem 2.1. Let $G(\mathbf{g}, \mathbf{a}, \mathbf{b})$ be a Bessel sequence in $L^{2}(\mathbb{R})$. Then $G(\mathbf{g}, \mathbf{a}, \mathbf{b})$ has Riesz property if and only if

$$
\operatorname{rank}\left(\Phi_{\mathbf{g}}(t, v)\right)=Q \text { for a.e. }(t, v) \in\left[0, \frac{1}{\beta q}\right) \times[0,1) .
$$


Proof. By Lemma 2.3, the equation (2.5) is equivalent to

$$
\operatorname{rank}\left(\Phi_{\mathbf{g}}(t, v)\right)=Q \text { for a.e. }(t, v) \in\left[0, \frac{1}{\beta}\right) \times[0,1) .
$$

So, to finish the proof, we only need to prove that $(2.6)$ is equivalent to $c=0$ being a unique solution to

$$
\overline{\Phi_{\mathbf{g}}^{*}(t, v)} c(t, v)=0 \text { for a.e. }(t, v) \in\left[0, \frac{1}{\beta}\right) \times[0,1)
$$

in $\left\{c=\left(c_{1}, c_{2}, \ldots, c_{L}\right): c_{l} \in l^{2}\left(\mathbb{Z}^{2}\right)\right.$ for $\left.1 \leq l \leq L\right\}$ by Lemmas 1.1 and 2.5. Suppose (2.6) holds. Then the columns of $\overline{\Phi_{\mathbf{g}}^{*}(t, v)}$ are linearly independent, which implies that $c=0$ is a unique solution to $(2.7)$.

Conversely, suppose $\operatorname{rank}\left(\Phi_{\mathbf{g}}(\cdot, \cdot)\right)<Q$ on some $E \subset\left[0, \frac{1}{\beta}\right) \times[0,1)$ with $|E|>0$. We denote by $\mathcal{P}(t, v)$ the orthogonal projection of $\mathbb{C}^{Q}$ onto $\operatorname{ker}\left(\Phi_{\mathrm{g}}^{*}(t\right.$, $v)$ ). Then there exists $i_{0} \in \mathbb{N}_{Q}$ such that $\mathcal{P}(\cdot, \cdot) \mathbf{e}_{i_{0}} \neq 0$ on some $E^{\prime} \subset E$ with $\left|E^{\prime}\right|>0$. Define $c=\left(c_{1}, c_{2}, \ldots, c_{L}\right)$ with $c_{l} \in l^{2}\left(\mathbb{Z}^{2}\right)$ by

$$
\overline{c(t, v)}= \begin{cases}\mathcal{P}(t, v) \mathbf{e}_{i_{0}} & \text { if }(t, v) \in E^{\prime} \\ 0, & \text { otherwise }\end{cases}
$$

for $(t, v) \in\left[0, \frac{1}{\beta}\right) \times[0,1)$. Then $c$ is well-defined by Lemma 2.6 and the fact that $\|c(t, v)\| \leq\left\|\mathbf{e}_{i_{0}}\right\|=1$ for a.e. $(t, v) \in\left[0, \frac{1}{\beta}\right) \times[0,1)$, and $c$ is a nonzero solution to (2.7). The proof is completed.

\section{Proofs of Theorems 1.1-1.3}

Proof of Theorem 1.1. Define

$$
\Gamma=\left\{f \in L^{2}(\mathbb{R}): \mathbf{Z}_{\alpha q} f \in L^{\infty}\left(\left[0, \frac{1}{\beta}\right) \times[0,1), \mathbb{C}^{p}\right)\right\} .
$$

Since $L^{\infty}\left(\left[0, \frac{1}{\beta}\right) \times[0,1), \mathbb{C}^{p}\right)$ is dense in $L^{2}\left(\left[0, \frac{1}{\beta}\right) \times[0,1), \mathbb{C}^{p}\right), \Gamma$ is dense in $L^{2}(\mathbb{R})$ by Lemma 1.1. So $G(\mathbf{g}, \mathbf{a}, \mathbf{b})$ is a frame for $L^{2}(\mathbb{R})$ with frame bounds $A$ and $B$ if and only if

$$
A\|f\|^{2} \leq \sum_{l=1}^{L} \sum_{m, n \in \mathbb{Z}}\left|\left\langle f, E_{m \beta} T_{n a_{l}} g_{l}\right\rangle\right|^{2} \leq B\|f\|^{2} \text { for } f \in \Gamma,
$$

i.e.,

$$
A\|f\|^{2} \leq \sum_{l=1}^{L} \sum_{\tau_{l}=0}^{\lambda_{l}-1} \sum_{r=0}^{q-1} \sum_{m, n \in \mathbb{Z}}\left|\left\langle f, E_{m \beta} T_{n \alpha q+r \alpha+\tau_{l} a_{l}} g_{l}\right\rangle\right|^{2} \leq B\|f\|^{2} \text { for } f \in \Gamma
$$

by the fact that $\mathbb{Z}=q \lambda_{l} \mathbb{Z}+\lambda_{l} \mathbb{N}_{q}+\mathbb{N}_{\lambda_{l}}$ for each $1 \leq l \leq L$. By Lemmas 1.1 and $2.2,(3.1)$ can be written as

$$
\beta A \int_{0}^{\frac{1}{\beta}} \int_{0}^{1}\left\|\mathbf{Z}_{\alpha q} f(t, v)\right\|^{2} d t d v
$$




$$
\begin{aligned}
& \leq \int_{0}^{\frac{1}{\beta}} \int_{0}^{1}\left\langle\Phi_{\mathbf{g}}^{*}(t, v) \Phi_{\mathbf{g}}(t, v) \overline{\mathbf{Z}_{\alpha q} f(t, v)}, \overline{\mathbf{Z}_{\alpha q} f(t, v)}\right\rangle d t v \\
& \leq \beta B \int_{0}^{\frac{1}{\beta}} \int_{0}^{1}\left\|\mathbf{Z}_{\alpha q} f(t, v)\right\|^{2} d t d v
\end{aligned}
$$

for $f \in \Gamma$. By Lemma 2.3,

$$
\beta A I \leq \Phi_{\mathbf{g}}^{*}(t, v) \Phi_{\mathbf{g}}(t, v) \leq \beta B I \text { for a.e. }(t, v) \in\left[0, \frac{1}{\beta q}\right) \times[0,1)
$$

if and only if

$$
\beta A I \leq \Phi_{\mathbf{g}}^{*}(t, v) \Phi_{\mathbf{g}}(t, v) \leq \beta B I \text { for a.e. }(t, v) \in\left[0, \frac{1}{\beta}\right) \times[0,1) .
$$

Therefore, to finish the proof, we only need to prove the equivalence between (3.2) and (3.3). It is obvious that (3.3) implies (3.2). Next we prove that (3.2) implies (3.3). Suppose (3.2) holds. Since each entry of $\Phi_{\mathbf{g}}^{*}(t, v) \Phi_{\mathbf{g}}(t, v)$ belongs to $L^{1}\left(\left[0, \frac{1}{\beta}\right) \times[0,1)\right)$, almost every point in $\left(0, \frac{1}{\beta}\right) \times(0,1)$ is a Lebesgue point of all entries of $\Phi_{\mathbf{g}}^{*}(t, v) \Phi_{\mathbf{g}}(t, v)$. Let $\left(t_{0}, v_{0}\right)$ be such a point. Choose $\epsilon>0$ such that $B\left(\left(t_{0}, v_{0}\right), \epsilon\right) \subset\left(0, \frac{1}{\beta}\right) \times(0,1)$. For $x \in \mathbb{C}^{p}$, define $f \in L^{2}(\mathbb{R})$ by

$$
\mathbf{Z}_{\alpha q} f(t, v)=\frac{\chi_{B\left(\left(t_{0}, v_{0}\right), \epsilon\right)}(t, v)}{\sqrt{\left|B\left(\left(t_{0}, v_{0}\right), \epsilon\right)\right|}} \bar{x} \text { for }(t, v) \in\left[0, \frac{1}{\beta}\right) \times[0,1) .
$$

Then $f \in \Gamma$. Applying (3.2) to such $f$, we have

$$
\beta A\|x\|^{2} \leq \frac{1}{\left|B\left(\left(t_{0}, v_{0}\right), \epsilon\right)\right|} \int_{B\left(\left(t_{0}, v_{0}\right), \epsilon\right)}\left\langle\Phi_{\mathbf{g}}^{*}(t, v) \Phi_{\mathbf{g}}(t, v) x, x\right\rangle \leq \beta B\|x\|^{2},
$$

and thus

$$
\beta A\|x\|^{2} \leq\left\langle\Phi_{\mathbf{g}}^{*}\left(t_{0}, v_{0}\right) \Phi_{\mathbf{g}}\left(t_{0}, v_{0}\right) x, x\right\rangle \leq \beta B\|x\|^{2}
$$

by letting $\epsilon \rightarrow 0$. This implies (3.3) by the arbitrariness of $x$ and $\left(t_{0}, v_{0}\right)$. The proof is completed.

Remark 3.1. By the proof of Theorem 1.1, $G(\mathbf{g}, \mathbf{a}, \mathbf{b})$ is a Bessel sequence in $L^{2}(\mathbb{R})$ with Bessel bound $B$ if and only if $\Phi_{\mathbf{g}}^{*}(t, v) \Phi_{\mathbf{g}}(t, v) \leq \beta B I$, equivalently, $\left\|\Phi_{\mathbf{g}}(t, v)\right\| \leq \sqrt{\beta B}$ for a.e. $(t, v) \in\left[0, \frac{1}{\beta q}\right) \times[0,1)$ when $\Phi_{\mathbf{g}}(t, v)$ is understood as an operator from $\mathbb{C}^{p}$ into $\mathbb{C}^{Q}$. It is well-known that such operator norm is equivalent to the one obtained by taking the maximum of the absolute values of all entries of a matrix. So $G(\mathbf{g}, \mathbf{a}, \mathbf{b})$ is a Bessel sequence in $L^{2}(\mathbb{R})$ if and only if $\mathcal{Z}_{\alpha q} g_{l} \in L^{\infty}\left(\mathbb{R}^{2}\right)$ for $1 \leq l \leq L$ by the quasi-periodicity of $\mathcal{Z}_{\alpha q}$.

Proof of Theorem 1.2. It is well-known that $G(\mathbf{g}, \mathbf{a}, \mathbf{b})$ is a Riesz basis for $L^{2}(\mathbb{R})$ with Riesz bounds $A$ and $B$ if and only if $G(\mathbf{g}, \mathbf{a}, \mathbf{b})$ has Riesz property and is a frame for $L^{2}(\mathbb{R})$ with frame bounds $A$ and $B$, and that $G(\mathbf{g}, \mathbf{a}, \mathbf{b})$ is an orthonormal basis for $L^{2}(\mathbb{R})$ if and only if it is a Riesz basis for $L^{2}(\mathbb{R})$ with Riesz bound 1. So we have Theorem 1.2 by Theorems 1.1 and 2.1. The proof is completed. 
Proof of Theorem 1.3. By Lemma 2.5, $G(\mathbf{h}, \mathbf{a}, \mathbf{b})$ is a dual of $G(\mathbf{g}, \mathbf{a}, \mathbf{b})$ if and only if, for each $f \in L^{2}(\mathbb{R})$,

$$
\mathbf{Z}_{\alpha q} f(t, v)=\overline{\Phi_{\mathbf{g}}^{*}(t, v)} d(t, v) \text { for a.e. }(t, v) \in\left[0, \frac{1}{\beta}\right) \times[0,1),
$$

where $d=\left(d_{1}, d_{2}, \ldots, d_{L}\right)$ with $d_{l, m, n}=\left\langle f, E_{m \beta} T_{n a_{l}} h_{l}\right\rangle$ for $1 \leq l \leq L$ and $m$, $n \in \mathbb{Z}$. Since $\mathbb{Z}=q \lambda_{l} \mathbb{Z}+\lambda_{l} \mathbb{N}_{q}+\mathbb{N}_{\lambda_{l}}$ (which implies that $a_{l} \mathbb{Z}=\alpha q \mathbb{Z}+\alpha \mathbb{N}_{q}+$ $a_{l} \mathbb{N}_{\lambda_{l}}$ ) for each $1 \leq l \leq L$, we have

$$
d_{l, m,(n q+r) \lambda_{l}+\tau_{l}}=\int_{0}^{\frac{1}{\beta}} \int_{0}^{1}\left(\overline{\mathcal{H}_{l}\left(t-\tau_{l} a_{l}, v\right)} \mathbf{Z}_{\alpha q} f(t, v)\right)_{r} e^{-2 \pi i m \beta t} e^{-2 \pi i n v} d t d v
$$

by Lemma 2.2 for $1 \leq l \leq L, r \in \mathbb{N}_{q}$ and $\tau_{l} \in \mathbb{N}_{\lambda_{l}}$. It follows that

$$
\sum_{m, n \in \mathbb{Z}} d_{l, m,(n q+r) \lambda_{l}+\tau_{l}} e^{2 \pi i m \beta t} e^{2 \pi i n v}=\frac{1}{\beta}\left(\overline{\mathcal{H}_{l}\left(t-\tau_{l} a_{l}, v\right)} \mathbf{Z}_{\alpha q} f(t, v)\right)_{r}
$$

for $1 \leq l \leq L, r \in \mathbb{N}_{q}$ and $\tau_{l} \in \mathbb{N}_{\lambda_{l}}$, equivalently, $d(t, v)=\frac{1}{\beta} \overline{\Phi_{\mathbf{h}}(t, v)} \mathbf{Z}_{\alpha q} f(t, v)$. So $G(\mathbf{h}, \mathbf{a}, \mathbf{b})$ is a dual of $G(\mathbf{g}, \mathbf{a}, \mathbf{b})$ if and only if

$$
\mathbf{Z}_{\alpha q} f(t, v)=\frac{1}{\beta} \overline{\Phi_{\mathbf{g}}^{*}(t, v) \Phi_{\mathbf{h}}(t, v)} \mathbf{Z}_{\alpha q} f(t, v)
$$

for $f \in L^{2}(\mathbb{R})$ and a.e. $(t, v) \in\left[0, \frac{1}{\beta}\right) \times[0,1)$ by $(3.4)$. By Lemma 2.3,

$$
\Phi_{\mathbf{g}}^{*}(t, v) \Phi_{\mathbf{h}}(t, v)=\beta I \text { for a.e. }(t, v) \in\left[0, \frac{1}{\beta q}\right) \times[0,1)
$$

if and only if

$$
\Phi_{\mathbf{g}}^{*}(t, v) \Phi_{\mathbf{h}}(t, v)=\beta I \text { for a.e. }(t, v) \in\left[0, \frac{1}{\beta}\right) \times[0,1) .
$$

Therefore, to finish the proof, we only need to prove the equivalence between (3.5) and (3.6). It is obvious that (3.6) implies (3.5). Next we prove the converse implication. Suppose (3.5) holds. For $k \in \mathbb{N}_{p}$, define $f \in L^{2}(\mathbb{R})$ by

$$
\mathbf{Z}_{\alpha q} f(t, v)=\mathbf{e}_{k} \text { for }(t, v) \in\left[0, \frac{1}{\beta}\right) \times[0,1) .
$$

Then it is well-defined by Lemma 1.1. Substituting such $f$ into (3.5), we have

$$
\mathbf{e}_{k}=\frac{1}{\beta} \overline{\Phi_{\mathbf{g}}^{*}(t, v) \Phi_{\mathbf{h}}(t, v)} \mathbf{e}_{k} \text { for a.e. }(t, v) \in\left[0, \frac{1}{\beta}\right) \times[0,1),
$$

which implies (3.6) by the arbitrariness of $k$. The proof is completed. 


\section{Examples}

This section is devoted to examples of Theorems 1.1-1.3. We first focus on the ones of Theorems 1.1 and 1.3.

Suppose

$$
\mathbf{a}=\left(\frac{1}{3}, \frac{2}{3}\right) \text { and } \mathbf{b}=(1,1) .
$$

Then $\alpha=\frac{p}{q}=\frac{2}{3}$ with $p=2, q=3$. For $\mathbf{g}=\left(g_{1}, g_{2}\right)$ with $g_{1}, g_{2} \in L^{2}(\mathbb{R})$, we associate it with $\Phi_{\mathbf{g}}$ as in Definition 1.1. Then

$$
\Phi_{\mathbf{g}}(t, v)=\left(\begin{array}{c}
G_{1}(t, v) \\
G_{2}(t, v)
\end{array}\right)
$$

with $G_{1}(t, v)=\left(\begin{array}{c}\mathcal{G}_{1}(t, v) \\ \mathcal{G}_{1}\left(t-\frac{1}{3}, v\right)\end{array}\right), G_{2}(t, v)=\mathcal{G}_{2}(t, v)$, where

$$
\mathcal{G}_{l}(t, v)=\left(\begin{array}{cc}
\mathcal{Z}_{2} g_{l}(t, v) & \mathcal{Z}_{2} g_{l}(t+1, v) \\
\mathcal{Z}_{2} g_{l}\left(t-\frac{2}{3}, v\right) & \mathcal{Z}_{2} g_{l}\left(t+\frac{1}{3}, v\right) \\
\mathcal{Z}_{2} g_{l}\left(t-\frac{4}{3}, v\right) & \mathcal{Z}_{2} g_{l}\left(t-\frac{1}{3}, v\right)
\end{array}\right)
$$

for $l=1,2$. By the quasi-periodicity of Zak transform, we have

$$
\mathcal{G}_{1}\left(t-\frac{1}{3}, v\right)=\left(\begin{array}{cc}
\mathcal{Z}_{2} g_{1}\left(t-\frac{1}{3}, v\right) & e^{-2 \pi i v} \mathcal{Z}_{2} g_{1}\left(t-\frac{4}{3}, v\right) \\
e^{2 \pi i v} \mathcal{Z}_{2} g_{1}(t+1, v) & \mathcal{Z}_{2} g_{1}(t, v) \\
e^{2 \pi i v} \mathcal{Z}_{2} g_{1}\left(t+\frac{1}{3}, v\right) & \mathcal{Z}_{2} g_{1}\left(t-\frac{2}{3}, v\right)
\end{array}\right) .
$$

So, for each $(t, v) \in \mathbb{R}^{2}, \mathcal{G}_{1}\left(t-\frac{1}{3}, v\right)$ is uniquely determined by $\mathcal{G}_{1}(t, v)$. Also observe that $\bigcup_{r=0}^{2} \bigcup_{k=0}^{1}\left(\left[0, \frac{1}{3}\right)+k-\frac{2}{3} r\right)$ is $2 \mathbb{Z}$-congruent to [0,2). It follows that $\mathbf{g}$ is uniquely determined by the values of $\Phi_{\mathbf{g}}(t, v)$ on $\left[0, \frac{1}{3}\right) \times[0,1)$. Therefore, an arbitrary matrix $9 \times 2$ matrix-valued function $\mathcal{K}(t, v)$ on $\left[0, \frac{1}{3}\right) \times[0,1)$ with all entries being in $L^{2}\left(\left[0, \frac{1}{3}\right) \times[0,1)\right)$ determines a unique $\mathbf{g}$ by

$$
\Phi_{\mathbf{g}}(t, v)=\mathcal{K}(t, v) \text { for a.e. }(t, v) \in\left[0, \frac{1}{3}\right) \times[0,1) .
$$

Let

$$
\mathcal{K}_{1}(t, v)=\left(\begin{array}{cc}
p(t) q_{1}(t) e^{2 \pi i j_{0} v} & 0 \\
p(t) q_{3}(t) e^{2 \pi i k_{0} v} & 0 \\
0 & p(t) q_{2}(t) e^{2 \pi i n_{0} v} \\
p(t) q_{2}(t) e^{2 \pi i n_{0} v} & 0 \\
0 & p(t) q_{1}(t) e^{2 \pi i j_{0} v} \\
0 & p(t) q_{3}(t) e^{2 \pi i k_{0} v} \\
\tilde{p}(t) \tilde{q}_{1}(t) e^{2 \pi i \tilde{j}_{0} v} & 0 \\
\tilde{p}(t) \tilde{q}_{3}(t) e^{2 \pi i \tilde{k}_{0} v} & 0 \\
0 & \tilde{p}(t) \tilde{q}_{2}(t) e^{2 \pi i \tilde{n}_{0} v}
\end{array}\right)
$$


for $(t, v) \in\left[0, \frac{1}{3}\right) \times[0,1)$, where $j_{0}, \tilde{j}_{0}, n_{0} \tilde{n}_{0}, k_{0}, \tilde{k}_{0} \in \mathbb{Z}$, and $p(t), \tilde{p}(t), q_{l}(t)$, $\tilde{q}_{l}(t)(l=1,2,3)$ are continuous functions about $t$ on $\left[0, \frac{1}{3}\right]$ satisfying

$$
\begin{aligned}
C_{1}(t)= & |p(t)|^{2}\left(\left|q_{1}(t)\right|^{2}+\left|q_{2}(t)\right|^{2}+\left|q_{3}(t)\right|^{2}\right) \\
& +|\tilde{p}(t)|^{2}\left(\left|\tilde{q}_{1}(t)\right|^{2}+\left|\tilde{q}_{3}(t)\right|^{2}\right) \neq 0, \\
C_{2}(t)= & |p(t)|^{2}\left(\left|q_{1}(t)\right|^{2}+\left|q_{2}(t)\right|^{2}+\left|q_{3}(t)\right|^{2}\right)+|\tilde{p}(t)|^{2}\left|\tilde{q}_{2}(t)\right|^{2} \neq 0
\end{aligned}
$$

for each $t \in\left[0, \frac{1}{3}\right]$. Define $\mathbf{g}=\left(g_{1}, g_{2}\right)$ by

$$
\Phi_{\mathbf{g}}(t, v)=\mathcal{K}_{1}(t, v) \text { for a.e. }(t, v) \in\left[0, \frac{1}{3}\right) \times[0,1) .
$$

Then we obtain the following example:

Example 4.1. Let $\mathbf{a}$ and $\mathbf{b}$ be defined as in (4.1), and let $\mathbf{g}=\left(g_{1}, g_{2}\right)$ be defined by

$$
g_{1}(t)= \begin{cases}p\left(t-2 j_{0}\right) q_{1}\left(t-2 j_{0}\right) & t \in\left[2 j_{0}, 2 j_{0}+\frac{1}{3}\right) ; \\ p\left(t+\frac{1}{3}-2 n_{0}\right) q_{2}\left(t+\frac{1}{3}-2 n_{0}\right) & t \in\left[-\frac{1}{3}+2 n_{0}, 2 n_{0}\right) ; \\ p\left(t+\frac{2}{3}-2 k_{0}\right) q_{3}\left(t+\frac{2}{3}-2 k_{0}\right) & t \in\left[-\frac{2}{3}+2 k_{0},-\frac{1}{3}+2 k_{0}\right) ; \\ 0 & \text { otherwise, }\end{cases}
$$

$$
g_{2}(t)= \begin{cases}\tilde{p}\left(t-2 \tilde{j}_{0}\right) \tilde{q}_{1}\left(t-2 \tilde{j}_{0}\right) & t \in\left[2 \tilde{j}_{0}, 2 \tilde{j}_{0}+\frac{1}{3}\right) ; \\ \tilde{p}\left(t+\frac{1}{3}-2 \tilde{n}_{0}\right) \tilde{q}_{2}\left(t+\frac{1}{3}-2 \tilde{n}_{0}\right) & t \in\left[-\frac{1}{3}+2 \tilde{n}_{0}, 2 \tilde{n}_{0}\right) ; \\ \tilde{p}\left(t+\frac{2}{3}-2 \tilde{k}_{0}\right) \tilde{q}_{3}\left(t+\frac{2}{3}-2 \tilde{k}_{0}\right) & t \in\left[-\frac{2}{3}+2 \tilde{k}_{0},-\frac{1}{3}+2 \tilde{k}_{0}\right) ; \\ 0 & \text { otherwise }\end{cases}
$$

for a.e. $t \in \mathbb{R}$, where $j_{0}, \tilde{j}_{0}, n_{0} \tilde{n}_{0}, k_{0}, \tilde{k}_{0} \in \mathbb{Z}$, and $p(t), \tilde{p}(t), q_{l}(t), \tilde{q}_{l}(t)(l=1$, $2,3)$ are continuous functions about $t$ on $\left[0, \frac{1}{3}\right]$ satisfying (4.7) and (4.8). Then $G(\mathbf{g}, \mathbf{a}, \mathbf{b})$ is a frame for $L^{2}(\mathbb{R})$.

Proof. By (4.6), (4.9) and the definition of Zak transform, we can easily obtain (4.10) and (4.11). And by the continuity of $p(t), \tilde{p}(t), q_{l}(t), \tilde{q}_{l}(t)(l=1,2,3)$, (4.7) and (4.8), we have

$$
\begin{aligned}
0<A & =\min \left\{C_{l}(t): l=1,2, t \in\left[0, \frac{1}{3}\right]\right\} \\
& \leq \max \left\{C_{l}(t): l=1,2, t \in\left[0, \frac{1}{3}\right]\right\}=B<\infty .
\end{aligned}
$$


It is easy to check that

$$
\left\langle\Phi_{\mathbf{g}}^{*}(t, v) \Phi_{\mathbf{g}}(t, v) x, x\right\rangle=C_{1}(t)\left|x_{1}\right|^{2}+C_{2}(t)\left|x_{2}\right|^{2}
$$

for $x \in \mathbb{C}^{2}$ and a.e. $(t, v) \in\left[0, \frac{1}{3}\right) \times[0,1)$. So

$$
A\|x\|^{2} \leq\left\langle\Phi_{\mathbf{g}}^{*}(t, v) \Phi_{\mathbf{g}}(t, v) x, x\right\rangle \leq B\|x\|^{2}
$$

for $x \in \mathbb{C}^{2}$ and a.e. $(t, v) \in\left[0, \frac{1}{3}\right) \times[0,1)$.

This implies that $G(\mathbf{g}, \mathbf{a}, \mathbf{b})$ is a frame for $L^{2}(\mathbb{R})$ with frame bounds $A$ and $B$ by Theorem 1.1.

Remark 4.1. In Example 4.1, by choosing good $p(t), \tilde{p}(t), q_{l}(t), \tilde{q}_{l}(t)(l=1,2$, 3 ) we can obtain a frame $G(\mathbf{g}, \mathbf{a}, \mathbf{b})$ with $g_{1}$ and $g_{2}$ having prescribed piecewise smoothness. In particular, if $k_{0}=n_{0}=j_{0}$ and $\tilde{k}_{0}=\tilde{n}_{0}=\tilde{j}_{0}$ in addition, we can obtain $g_{1}$ and $g_{2}$ having prescribed smoothness on the whole line $\mathbb{R}$ by a careful choice of $p(t), \tilde{p}(t), q_{l}(t), \tilde{q}_{l}(t)(l=1,2,3)$. Let us illustrate this by the following arguments. Suppose $k_{0}=n_{0}=j_{0}=j$ and $\tilde{k}_{0}=\tilde{n}_{0}=\tilde{j}_{0}=\tilde{j}$ in Example 4.1. Take continuous functions $p(t), \tilde{p}(t), q_{l}(t), \tilde{q}_{l}(t)(l=1,2,3)$ on $\left[0, \frac{1}{3}\right]$ satisfying $(4.7),(4.8)$ and

$$
\begin{aligned}
& q_{3}(0)=q_{1}\left(\frac{1}{3}\right)=0, p\left(\frac{1}{3}\right) q_{3}\left(\frac{1}{3}\right)=p(0) q_{2}(0), p\left(\frac{1}{3}\right) q_{2}\left(\frac{1}{3}\right)=p(0) q_{1}(0) \\
& \tilde{q}_{3}(0)=\tilde{q}_{1}\left(\frac{1}{3}\right)=0, \tilde{p}\left(\frac{1}{3}\right) \tilde{q}_{3}\left(\frac{1}{3}\right)=\tilde{p}(0) \tilde{q}_{2}(0), \tilde{p}\left(\frac{1}{3}\right) \tilde{q}_{2}\left(\frac{1}{3}\right)=\tilde{p}(0) \tilde{q}_{1}(0) .
\end{aligned}
$$

Then both $g_{1}$ and $g_{2}$ are continuous on $\mathbb{R}$, and $G(\mathbf{g}, \mathbf{a}, \mathbf{b})$ is a frame for $L^{2}(\mathbb{R})$ by Example 4.1. If we make the following four assumptions, then both $g_{1}$ and $g_{2}$ have continuous derivatives of order one on $\mathbb{R}$, and $G(\mathbf{g}, \mathbf{a}, \mathbf{b})$ is a frame for $L^{2}(\mathbb{R})$ by Example 4.1:

(I) $p(t), \tilde{p}(t), q_{l}(t), \tilde{q}_{l}(t)(l=1,2,3)$ have continuous derivatives of order one on $\left[0, \frac{1}{3}\right]$;

(II) $(4.7),(4.8),(4.12),(4.13)$ hold;

(III)

$$
\begin{aligned}
p(t) q_{1}(t)= & 2 p(0) q_{1}(0)-p\left(\frac{1}{3}-t\right) q_{2}\left(\frac{1}{3}-t\right) \\
& \text { for } t \in\left[0, \delta_{1}\right] \text { with some } 0<\delta_{1}<\frac{1}{6} ; \\
p(t) q_{2}(t)= & 2 p(0) q_{2}(0)-p\left(\frac{1}{3}-t\right) q_{3}\left(\frac{1}{3}-t\right) \\
& \text { for } t \in\left[0, \delta_{2}\right] \text { with some } 0<\delta_{2}<\frac{1}{6} ;
\end{aligned}
$$

(IV)

$$
\begin{aligned}
\tilde{p}(t) \tilde{q}_{1}(t)= & 2 \tilde{p}(0) \tilde{q}_{1}(0)-\tilde{p}\left(\frac{1}{3}-t\right) \tilde{q}_{2}\left(\frac{1}{3}-t\right) \\
& \text { for } t \in\left[0, \tilde{\delta}_{1}\right] \text { with some } 0<\tilde{\delta}_{1}<\frac{1}{6}
\end{aligned}
$$




$$
\begin{aligned}
\tilde{p}(t) \tilde{q}_{2}(t)= & 2 \tilde{p}(0) \tilde{q}_{2}(0)-\tilde{p}\left(\frac{1}{3}-t\right) \tilde{q}_{3}\left(\frac{1}{3}-t\right) \\
& \text { for } t \in\left[0, \tilde{\delta}_{2}\right] \text { with some } 0<\tilde{\delta}_{2}<\frac{1}{6} .
\end{aligned}
$$

Now let

$$
\mathcal{K}_{2}(t, v)=\left(\begin{array}{cc}
m_{0}(v) & 0 \\
m_{2}(v) & 0 \\
0 & m_{1}(v) \\
m_{1}(v) & 0 \\
0 & m_{0}(v) \\
0 & m_{2}(v) \\
\tilde{m}_{1}(v) & 0 \\
0 & \tilde{m}_{0}(v) \\
0 & \tilde{m}_{2}(v)
\end{array}\right)
$$

for $(t, v) \in\left[0, \frac{1}{3}\right) \times[0,1)$, where $m_{l}(v), \tilde{m}_{l}(v)(l=0,1,2)$ are continuous functions about $v$ on $[0,1]$ satisfying

$$
\begin{aligned}
& D_{1}(v)=\left|m_{0}(v)\right|^{2}+\left|m_{1}(v)\right|^{2}+\left|m_{2}(v)\right|^{2}+\left|\tilde{m}_{1}(v)\right|^{2} \neq 0 \\
& D_{2}(v)=\left|m_{0}(v)\right|^{2}+\left|m_{1}(v)\right|^{2}+\left|m_{2}(v)\right|^{2}+\left|\tilde{m}_{0}(v)\right|^{2}+\left|\tilde{m}_{2}(v)\right|^{2} \neq 0
\end{aligned}
$$

for each $v \in[0,1]$. Put $A=\min \left\{D_{l}(v): l=1,2, v \in[0,1]\right\}, B=\max \left\{D_{l}(v)\right.$ : $l=1,2, v \in[0,1]\}$. Define $\mathbf{g}=\left(g_{1}, g_{2}\right)$ by

$$
\Phi_{\mathbf{g}}(t, v)=\mathcal{K}_{2}(t, v) .
$$

Then, by an argument similar to Example 4.1, we have the following example:

Example 4.2. Let $\mathbf{a}$ and $\mathbf{b}$ be defined as in (4.1). Define $\mathbf{g}=\left(g_{1}, g_{2}\right)$ as in (4.16). Then $G(\mathbf{g}, \mathbf{a}, \mathbf{b})$ is a frame for $L^{2}(\mathbb{R})$ with frame bounds $A$ and $B$.

Next we give two explicit expressions of $\mathbf{g}=\left(g_{1}, g_{2}\right)$ in Example 4.2. Observe that, for $\mu_{1}, \mu_{2}>0$, we always have

$$
\begin{aligned}
\left(\mu_{1} \cos ^{2} 2 \pi v+\mu_{2} \sin ^{2} 2 \pi v\right)^{N} & =\sum_{k=0}^{N}\left[\sqrt{C_{N}^{k}}\left(\sqrt{\mu_{1}} \cos 2 \pi v\right)^{N-k}\left(\sqrt{\mu_{2}} \sin 2 \pi v\right)^{k}\right]^{2} \\
& >0
\end{aligned}
$$

Motivated by this fact, we give two concrete constructions of $\mathbf{g}$.

(1) Let $N=4$ in (4.17), and choose $m_{l}(v), \tilde{m}_{l}(v)(l=0, l, 2)$ in (4.16) as follows:

$$
\begin{aligned}
& m_{0}(v)=\mu_{1}^{2} \cos ^{4} 2 \pi v, m_{1}(v)=i 2 \mu_{1}^{\frac{3}{2}} \mu_{2}^{\frac{1}{2}} \cos ^{3} 2 \pi v \sin 2 \pi v \\
& m_{2}(v)=\sqrt{6} \mu_{1} \mu_{2} \cos ^{2} 2 \pi v \sin ^{2} 2 \pi v \\
& \tilde{m}_{0}(v)=i 2 \mu_{1}^{\frac{1}{2}} \mu_{2}^{\frac{3}{2}} \cos 2 \pi v \sin ^{3} 2 \pi v \\
& \tilde{m}_{1}(v)=\tilde{m}_{2}(v)=\mu_{2}^{2} \sin ^{4} 2 \pi v
\end{aligned}
$$


Then $D_{1}(v)$ and $D_{2}(v)$ are both continuous on $[0,1]$, and

$$
\begin{aligned}
& D_{1}(v) \geq \mu_{1}^{4} \cos ^{8} 2 \pi v+\mu_{2}^{4} \sin ^{8} 2 \pi v>0, \\
& D_{2}(v)=\left(\mu_{1} \cos ^{2} 2 \pi v+\mu_{2} \sin ^{2} 2 \pi v\right)^{4}>0
\end{aligned}
$$

for $v \in[0,1]$. So (4.14) and (4.15) hold. By Example 4.2, we obtain a frame $G(\mathbf{g}, \mathbf{a}, \mathbf{b})$ for $L^{2}(\mathbb{R})$, where

$$
\begin{aligned}
g_{1}(t)= & \frac{3}{8} \mu_{1}^{2} \chi_{\left[0, \frac{1}{3}\right)}(t)+\frac{1}{4} \mu_{1}^{2} \chi_{\left[-4,-\frac{11}{3}\right) \cup\left[4, \frac{13}{3}\right)}(t)+\frac{1}{16} \mu_{1}^{2} \chi_{\left[-8,-\frac{23}{3}\right) \cup\left[8, \frac{25}{3}\right)}(t) \\
& +\frac{1}{4} \mu_{1}^{\frac{3}{2}} \mu_{2}^{\frac{1}{2}} \chi_{\left[\frac{11}{3}, 4\right)}(t)-\frac{1}{4} \mu_{1}^{\frac{3}{2}} \mu_{2}^{\frac{1}{2}} \chi_{\left[-\frac{13}{3},-4\right)}(t)+\frac{1}{8} \mu_{1}^{\frac{3}{2}} \mu_{2}^{\frac{1}{2}} \chi_{\left[\frac{23}{3}, 8\right)}(t) \\
& -\frac{1}{8} \mu_{1}^{\frac{3}{2}} \mu_{2}^{\frac{1}{2}} \chi_{\left[-\frac{25}{3},-8\right)}(t)+\frac{\sqrt{6}}{8} \mu_{1} \mu_{2} \chi_{\left[-\frac{2}{3},-\frac{1}{3}\right)}(t) \\
& -\frac{\sqrt{6}}{16} \mu_{1} \mu_{2} \chi_{\left[-\frac{26}{3},-\frac{25}{3}\right) \cup\left[\frac{22}{3}, \frac{23}{3}\right)}(t), \\
g_{2}(t)= & \frac{3}{8} \mu_{2}^{2} \chi_{\left[-\frac{1}{3}, \frac{1}{3}\right)}(t)-\frac{1}{4} \mu_{2}^{2} \chi_{\left[\frac{11}{3}, \frac{13}{3}\right) \cup\left[-\frac{13}{3},-\frac{11}{3}\right)}(t)+\frac{1}{16} \mu_{2}^{2} \chi_{\left[-\frac{25}{3},-\frac{23}{3}\right) \cup\left[\frac{23}{3}, \frac{25}{3}\right)}(t) \\
& +\frac{1}{4} \mu_{1}^{\frac{1}{2}} \mu_{2}^{\frac{3}{2}} \chi_{\left[\frac{13}{3}, \frac{14}{3}\right)}(t)-\frac{1}{4} \mu_{1}^{\frac{1}{2}} \mu_{2}^{\frac{3}{2}} \chi_{\left[-\frac{11}{3},-\frac{10}{3}\right)}(t)-\frac{1}{8} \mu_{1}^{\frac{1}{2}} \mu_{2}^{\frac{3}{2}} \chi_{\left[\frac{25}{3}, \frac{26}{3}\right)}(t) \\
& +\frac{1}{8} \mu_{1}^{\frac{1}{2}} \mu_{2}^{\frac{3}{2}} \chi_{\left[-\frac{23}{3},-\frac{22}{3}\right)}(t) .
\end{aligned}
$$

(2) Let $N=3$, and choose $m_{l}(v), \tilde{m}_{l}(v)(l=0, l, 2)$ in (4.16) as follows:

$$
\begin{aligned}
& m_{0}(v)=\mu_{1}^{\frac{3}{2}} \cos ^{3} 2 \pi v, m_{1}(v)=i \sqrt{3} \mu_{1} \mu_{2}^{\frac{1}{2}} \cos ^{2} 2 \pi v \sin 2 \pi v, \\
& m_{2}(v)=\sqrt{3} \mu_{1}^{\frac{1}{2}} \mu_{2} \cos 2 \pi v \sin ^{2} 2 \pi v, \\
& \tilde{m}_{1}(v)=i \mu_{2}^{\frac{3}{2}} \sin ^{3} 2 \pi v, \\
& \tilde{m}_{0}(v)=i \beta_{0} \mu_{2}^{\frac{3}{2}} \sin ^{3} 2 \pi v, \\
& \tilde{m}_{2}(v)=i \beta_{2} \mu_{2}^{\frac{3}{2}} \sin ^{3} 2 \pi v,
\end{aligned}
$$

where $\beta_{0}, \beta_{2} \geq 0$, and $\beta_{0}^{2}+\beta_{2}^{2}=1$. Then

$$
D_{1}(v)=D_{2}(v)=\left(\mu_{1} \cos ^{2} 2 \pi v+\mu_{2} \sin ^{2} 2 \pi v\right)^{3}>0
$$

for $v \in[0,1]$. So (4.14) and (4.15) hold, and Example 4.2 provides us with a frame $G(\mathbf{g}, \mathbf{a}, \mathbf{b})$ for $L^{2}(\mathbb{R})$, where

$$
\begin{aligned}
g_{1}(t)= & \frac{3}{8} \mu_{1}^{\frac{3}{2}} \chi_{\left[-2,-\frac{5}{3}\right) \cup\left[2, \frac{7}{3}\right)}(t)+\frac{1}{8} \mu_{1}^{\frac{3}{2}} \chi_{\left[-6,-\frac{17}{3}\right) \cup\left[6, \frac{19}{3}\right)}(t)+\frac{\sqrt{3}}{8} \mu_{1} \mu_{2}^{\frac{1}{2}} \chi_{\left[\frac{5}{3}, 2\right) \cup\left[\frac{17}{3}, 6\right)}(t) \\
& -\frac{\sqrt{3}}{8} \mu_{1} \mu_{2}^{\frac{1}{2}} \chi_{\left[-\frac{19}{3},-6\right) \cup\left[-\frac{7}{3},-2\right)}(t)+\frac{\sqrt{3}}{8} \mu_{1}^{\frac{1}{2}} \mu_{2} \chi_{\left[\frac{4}{3}, \frac{5}{3}\right) \cup\left[-\frac{8}{3},-\frac{7}{3}\right)}(t) \\
& -\frac{\sqrt{3}}{8} \mu_{1}^{\frac{1}{2}} \mu_{2} \chi_{\left[-\frac{20}{3},-\frac{19}{3}\right) \cup\left[\frac{16}{3}, \frac{17}{3}\right)}(t), \\
g_{2}(t)= & \frac{3}{8} \mu_{2}^{\frac{3}{2}} \chi_{\left[2, \frac{7}{3}\right)}(t)-\frac{3}{8} \mu_{2}^{\frac{3}{2}} \chi_{\left[-2,-\frac{5}{3}\right)}(t)+\frac{1}{8} \mu_{2}^{\frac{3}{2}} \chi_{\left[-6,-\frac{17}{3}\right)}(t)-\frac{1}{8} \mu_{2}^{\frac{3}{2}} \chi_{\left[6, \frac{19}{3}\right)}(t)
\end{aligned}
$$




$$
\begin{aligned}
& +\frac{3}{8} \beta_{0} \mu_{2}^{\frac{3}{2}} \chi_{\left[\frac{7}{3}, \frac{8}{3}\right)}(t)-\frac{3}{8} \beta_{0} \mu_{2}^{\frac{3}{2}} \chi_{\left[-\frac{5}{3},-\frac{4}{3}\right)}(t)-\frac{1}{8} \beta_{0} \mu_{2}^{\frac{3}{2}} \chi_{\left[\frac{19}{3}, \frac{20}{3}\right)}(t) \\
& +\frac{1}{8} \beta_{0} \mu_{2}^{\frac{3}{2}} \chi_{\left[-\frac{17}{3},-\frac{16}{3}\right)}(t)+\frac{3}{8} \beta_{2} \mu_{2}^{\frac{3}{2}} \chi_{\left[\frac{5}{3}, \frac{6}{3}\right)}(t)-\frac{3}{8} \beta_{2} \mu_{2}^{\frac{3}{2}} \chi_{\left[-\frac{7}{3},-2\right)}(t) \\
& +\frac{1}{8} \beta_{2} \mu_{2}^{\frac{3}{2}} \chi_{\left[-\frac{19}{3},-6\right)}(t)-\frac{1}{8} \beta_{2} \mu_{2}^{\frac{3}{2}} \chi_{\left[\frac{17}{3}, 6\right)}(t) .
\end{aligned}
$$

Now we turn to Theorem 1.3. We still work under (4.1). Let $\mathbf{g}=\left(g_{1}, g_{2}\right)$ be associated with $\Phi_{\mathbf{g}}$ as in (4.2), and $\mathbf{h}=\left(h_{1}, h_{2}\right)$ with $\Phi_{\mathbf{h}}$ similarly. A simple computation shows that

$$
G_{1}^{*}(t, v) H_{1}(t, v)=\left(\begin{array}{cc}
A_{1}(t, v) & B_{1}(t, v) \\
e^{2 \pi i v} B_{1}(t, v) & A_{1}(t, v)
\end{array}\right),
$$

where

$$
\begin{aligned}
A_{1}(t, v)= & \overline{\mathcal{Z}_{2} g_{1}(t, v)} \mathcal{Z}_{2} h_{1}(t, v)+\overline{\mathcal{Z}_{2} g_{1}\left(t-\frac{2}{3}, v\right)} \mathcal{Z}_{2} h_{1}\left(t-\frac{2}{3}, v\right) \\
& +\overline{\mathcal{Z}_{2} g_{1}\left(t-\frac{4}{3}, v\right)} \mathcal{Z}_{2} h_{1}\left(t-\frac{4}{3}, v\right)+\overline{\mathcal{Z}_{2} g_{1}\left(t-\frac{1}{3}, v\right)} \mathcal{Z}_{2} h_{1}\left(t-\frac{1}{3}, v\right) \\
& +\overline{\mathcal{Z}_{2} g_{1}(t+1, v)} \mathcal{Z}_{2} h_{1}(t+1, v)+\overline{\mathcal{Z}_{2} g_{1}\left(t+\frac{1}{3}, v\right)} \mathcal{Z}_{2} h_{1}\left(t+\frac{1}{3}, v\right), \\
B_{1}(t, v)= & \overline{\mathcal{Z}_{2} g_{1}(t, v)} \mathcal{Z}_{2} h_{1}(t+1, v)+\overline{\mathcal{Z}_{2} g_{1}\left(t-\frac{2}{3}, v\right)} \mathcal{Z}_{2} h_{1}\left(t+\frac{1}{3}, v\right) \\
& +\overline{\mathcal{Z}_{2} g_{1}\left(t-\frac{4}{3}, v\right)} \mathcal{Z}_{2} h_{1}\left(t-\frac{1}{3}, v\right)+e^{-2 \pi i v} \overline{\mathcal{Z}_{2} g_{1}\left(t-\frac{1}{3}, v\right)} \mathcal{Z}_{2} h_{1}\left(t-\frac{4}{3}, v\right) \\
+ & e^{-2 \pi i v} \overline{\mathcal{Z}_{2} g_{1}(t+1, v)} \mathcal{Z}_{2} h_{1}(t, v)+e^{-2 \pi i v} \overline{\mathcal{Z}_{2} g_{1}\left(t+\frac{1}{3}, v\right)} \mathcal{Z}_{2} h_{1}\left(t-\frac{2}{3}, v\right) .
\end{aligned}
$$

It follows that

$$
\Phi_{\mathbf{g}}^{*}(t, v) \Phi_{\mathbf{h}}(t, v)=I \text { for a.e. }(t, v) \in\left[0, \frac{1}{3}\right) \times[0,1)
$$

if and only if

$$
G_{2}^{*}(t, v) H_{2}(t, v)=\left(\begin{array}{cc}
1-A_{1}(t, v) & -B_{1}(t, v) \\
-e^{2 \pi i v} B_{1}(t, v) & 1-A_{1}(t, v)
\end{array}\right)
$$

for a.e. $(t, v) \in\left[0, \frac{1}{3}\right) \times[0,1)$.

In particular, (4.19) can be written as

$$
G_{2}^{*}(t, v) H_{2}(t, v)=\left(1-A_{1}(t, v)\right) I \text { for a.e. }(t, v) \in\left[0, \frac{1}{3}\right) \times[0,1)
$$

if

$$
B_{1}(t, v)=0 \text { for a.e. }(t, v) \in\left[0, \frac{1}{3}\right) \times[0,1)
$$


Now suppose (4.21) holds, and $\tilde{g}_{2}, \tilde{h}_{2} \in L^{2}(\mathbb{R})$ with $\mathcal{Z}_{2} \tilde{g}_{2}, \mathcal{Z}_{2} \tilde{h}_{2} \in L^{\infty}\left(\mathbb{R}^{2}\right)$ are such that

$$
\tilde{G}_{2}^{*}(t, v) \tilde{H}_{2}(t, v)=I \text { for a.e. }(t, v) \in\left[0, \frac{1}{3}\right) \times[0,1) .
$$

Define $g_{2}$ and $h_{2}$ by

$$
\begin{aligned}
& G_{2}(t, v)=C(t, v) \tilde{G}_{2}(t, v), \\
& H_{2}(t, v)=D(t, v) \tilde{H}_{2}(t, v),
\end{aligned}
$$

where $C(t, v), D(t, v) \in L^{\infty}\left(\left[0, \frac{1}{3}\right) \times[0,1)\right)$, and $\overline{C(t, v)} D(t, v)=1-A_{1}(t, v)$ for a.e. $(t, v) \in\left[0, \frac{1}{3}\right) \times[0,1)$. Then (4.18) holds, and thus $G(\mathbf{g}, \mathbf{a}, \mathbf{b})$ and $G(\mathbf{h}, \mathbf{a}, \mathbf{b})$ are a pair of dual frames by Theorem 1.3. It is easy to design $g_{1}, h_{1}$ and $g_{2}, h_{2}$ satisfying (4.21)-(4.24). Indeed, UEP method by [17] can be used to construct $\tilde{g}_{2}=\tilde{h}_{2}$ satisfying (4.22). Now we collect our arguments as the following example:

Example 4.3. Let $\mathbf{a}$ and $\mathbf{b}$ be defined as in (4.1). Define $\mathbf{g}=\left(g_{1}, g_{2}\right)$ and $\mathbf{h}=\left(h_{1}, h_{2}\right)$ such that $\mathcal{Z}_{2} g_{l}, \mathcal{Z}_{2} h_{l} \in L^{\infty}\left(\mathbb{R}^{2}\right)$ for $l=1,2$, and that (4.21) -(4.24) hold. Then $G(\mathbf{g}, \mathbf{a}, \mathbf{b})$ and $G(\mathbf{h}, \mathbf{a}, \mathbf{b})$ are a pair of dual frames.

Next we give a special case of Example 4.3 in terms of orthogonal filters. We know that there are many such filters. So it shows that we have much freedom to design many different pairs of Gabor dual frames.

Example 4.4. In Example 4.3, define $g_{1}$ and $h_{1}$ by $G_{1}(t, v)$ and $H_{1}(t, v)$ such that

$$
\begin{aligned}
& \mathcal{Z}_{2} g_{1}(t, v)=\mathcal{Z}_{2} h_{1}(t, v)=q_{0,0}(t, v)=1, \\
& \mathcal{Z}_{2} g_{1}(t+1, v)=-e^{-2 \pi i v} \overline{\mathcal{Z}_{2} h_{1}(t+1, v)}=p(t, v) q_{0,1}(t, v), \\
& \mathcal{Z}_{2} g_{1}\left(t-\frac{2}{3}, v\right)=\overline{\mathcal{Z}_{2} h_{1}\left(t-\frac{2}{3}, v\right)}=p(t, v) q_{1,0}(t, v), \\
& \mathcal{Z}_{2} g_{1}\left(t+\frac{1}{3}, v\right)=-e^{-2 \pi i v} \frac{\mathcal{Z}_{2} h_{1}\left(t+\frac{1}{3}, v\right)}{\mathcal{Z}_{2} h_{1}\left(t-\frac{4}{3}, v\right)}=p(t, v) q_{1,1}(t, v), \\
& \left.\mathcal{Z}_{2} g_{1}\left(t-\frac{4}{3}, v\right)=p\right) q_{2,0}(t, v), \\
& \mathcal{Z}_{2} g_{1}\left(t-\frac{1}{3}, v\right)=-e^{-2 \pi i v} \frac{\mathcal{Z}_{2} h_{1}\left(t-\frac{1}{3}, v\right)}{\mathcal{Z}}=p(t, v) q_{2,1}(t, v)
\end{aligned}
$$

for a.e. $(t, v) \in\left[0, \frac{1}{3}\right) \times[0,1)$, where $p(t, v), q_{i, j}(t, v) \in L^{\infty}\left(\left[0, \frac{1}{3}\right) \times[0,1)\right)$ for $(i, j) \in\{0,1,2\} \times\{0,1\}$. Suppose $m(v)$ is an orthogonal filter, i.e., $m(v)$ is a $\mathbb{Z}$-periodic measurable function satisfying $|m(v)|^{2}+\left|m\left(v+\frac{1}{2}\right)\right|^{2}=1$ for a.e. $v \in \mathbb{R}$. Let $\tilde{g}_{2}, \tilde{h}_{2}$ be defined by

$$
\tilde{G}_{2}(t, v)=\tilde{H}_{2}(t, v)=\left(\begin{array}{cc}
m(v) & e^{-2 \pi i v} \overline{m\left(v+\frac{1}{2}\right)} \\
0 & 0 \\
m\left(v+\frac{1}{2}\right) & -e^{-2 \pi i v} \overline{m(v)}
\end{array}\right)=M(v)
$$


for a.e. $(t, v) \in\left[0, \frac{1}{3}\right) \times[0,1)$. Define $g_{2}$ and $h_{2}$ by $G_{2}(t, v)$ and $H_{2}(t, v)$ such that

$$
\begin{aligned}
& G_{2}(t, v)=(p(t, v))^{2} M(v), \\
& H_{2}(t, v)=\left[e^{-2 \pi i v}\left(\overline{\left(\overline{q_{0,1}(t, v)}\right)^{2}}+\left(\overline{q_{1,1}(t, v)}\right)^{2}+\left(\overline{q_{2,1}(t, v)}\right)^{2}\right)\right. \\
& \left.-\left(\overline{q_{1,0}(t, v)}\right)^{2}-\left(\overline{q_{2,0}(t, v)}\right)^{2}\right] M(v) .
\end{aligned}
$$

Then $G(\mathbf{g}, \mathbf{a}, \mathbf{b})$ and $G(\mathbf{h}, \mathbf{a}, \mathbf{b})$ are a pair of dual frames by Example 4.3.

Finally, we give an example of Theorem 1.2. Suppose

$$
\mathbf{a}=\left(\frac{3}{2}, 3\right) \text { and } \mathbf{b}=(1,1) \text {. }
$$

Then $\alpha=\frac{p}{q}=3, \lambda_{1}=2, \lambda_{2}=1$, and $Q=\left(\lambda_{1}+\lambda_{2}\right) q=p=3$. For $\mathbf{g}=\left(g_{1}, g_{2}\right)$, we associate it with $\Phi_{\mathbf{g}}(t, v)$ as defined as in Definition 1.1, where

$\Phi_{\mathbf{g}}(t, v)=\left(\begin{array}{c}G_{1}(t, v) \\ G_{2}(t, v)\end{array}\right)$ with $G_{1}(t, v)=\left(\begin{array}{c}\mathcal{G}_{1}(t, v) \\ \mathcal{G}_{1}\left(t-\frac{3}{2}, v\right)\end{array}\right), G_{2}(t, v)=\mathcal{G}_{2}(t, v)$,

and

$$
\begin{aligned}
& \mathcal{G}_{l}(t, v)=\left(\mathcal{Z}_{3} g_{l}(t, v), \mathcal{Z}_{3} g_{l}(t+1, v), \mathcal{Z}_{3} g_{l}(t+2, v)\right) \text { for } l=1,2 \\
& \mathcal{G}_{1}\left(t-\frac{3}{2}, v\right)=\left(\mathcal{Z}_{3} g_{1}\left(t-\frac{3}{2}, v\right), \mathcal{Z}_{3} g_{1}\left(t-\frac{1}{2}, v\right), \mathcal{Z}_{3} g_{1}\left(t+\frac{1}{2}, v\right)\right)
\end{aligned}
$$

It is obvious that $g_{2}$ is completely determined by the values of $\mathcal{G}_{2}(t, v)$ on $[0,1) \times[0,1)$. Observe that $\bigcup_{k \in \mathbb{N}_{3}}\left(\left[0, \frac{1}{2}\right) \cup\left[-\frac{3}{2},-1\right)+k\right)$ is $3 \mathbb{Z}$-congruent to $[0,3)$. This implies that the values of $G_{1}(t, v)$ on $\left[0, \frac{1}{2}\right) \times[0,1)$ determine the ones of $G_{1}(t, v)$ on $\left[\frac{1}{2}, 1\right) \times[0,1)$, and thus $g_{1}$ is completely determined by the $G_{1}(t, v)$ on $\left[0, \frac{1}{2}\right) \times[0,1)$. By the quasi-periodicity of Zak transform, we have

$$
\begin{aligned}
G_{1}\left(t+\frac{1}{2}, v\right) & =\left(\begin{array}{ccc}
\mathcal{Z}_{3} g_{1}\left(t+\frac{1}{2}, v\right) & \mathcal{Z}_{3} g_{1}\left(t+\frac{1}{2}+1, v\right) & \mathcal{Z}_{3} g_{1}\left(t+\frac{1}{2}+2, v\right) \\
\mathcal{Z}_{3} g_{1}\left(t+\frac{1}{2}-\frac{3}{2}, v\right) & \mathcal{Z}_{3} g_{1}(t, v) & \mathcal{Z}_{3} g_{1}(t+1, v)
\end{array}\right) \\
& =\left(\begin{array}{ccc}
\mathcal{Z}_{3} g_{1}\left(t+\frac{1}{2}, v\right) & e^{-2 \pi i v} \mathcal{Z}_{3} g_{1}\left(t-\frac{3}{2}, v\right) & e^{-2 \pi i v} \mathcal{Z}_{3} g_{1}\left(t-\frac{1}{2}, v\right) \\
e^{2 \pi i v} \mathcal{Z}_{3} g_{1}(t+2, v) & \mathcal{Z}_{3} g_{1}(t, v) & \mathcal{Z}_{3} g_{1}(t+1, v)
\end{array}\right)
\end{aligned}
$$

for $(t, v) \in\left[0, \frac{1}{2}\right) \times[0,1)$. Let

$$
\mathcal{K}_{3}(t, v)=\left\{\begin{array}{c}
\left(\begin{array}{ccc}
p_{0}(t, v) & 0 & p_{1}(t, v) \\
0 & p_{2}(t, v) & 0 \\
\tilde{p}_{0}(t, v) & 0 & \tilde{p}_{1}(t, v)
\end{array}\right) \\
0 \\
\left(\begin{array}{ccc}
0 & 0 & p_{2}\left(t-\frac{1}{2}, v\right) e^{-2 \pi i v} \\
p_{1}\left(t-\frac{1}{2}, v\right) e^{2 \pi i v} & p_{0}\left(t-\frac{1}{2}, v\right) & 0 \\
\tilde{p}_{2}(t, v) & \tilde{p}_{3}(t, v) & 0
\end{array}\right) \text { for }(t, v) \in\left[0, \frac{1}{2}\right) \times[0,1) ;
\end{array}\right.
$$

where $p_{l}(t, v), \tilde{p}_{l^{\prime}}(t, v)\left(l=0,1,2, l^{\prime}=0,1\right)$ are continuous functions on $\left[0, \frac{1}{2}\right] \times[0,1]$, and $\tilde{p}_{2}(t, v), \tilde{p}_{3}(t, v)$ are continuous on $\left[\frac{1}{2}, 1\right] \times[0,1]$ satisfying

$$
\begin{aligned}
& P_{0}(t, v)=\left|p_{0}(t, v)\right|^{2}+\left|\tilde{p}_{0}(t, v)\right|^{2} \neq 0, \\
& P_{1}(t, v)=\left|p_{1}(t, v)\right|^{2}+\left|\tilde{p}_{1}(t, v)\right|^{2} \neq 0,
\end{aligned}
$$




$$
\begin{aligned}
& P_{2}(t, v)=\left|p_{2}(t, v)\right|^{2} \neq 0, \\
& P_{3}(t, v)=\left|\tilde{p}_{2}\left(t+\frac{1}{2}, v\right)\right|^{2}+\left|p_{1}(t, v)\right|^{2} \neq 0, \\
& P_{4}(t, v)=\left|\tilde{p}_{3}\left(t+\frac{1}{2}, v\right)\right|^{2}+\left|p_{0}(t, v)\right|^{2} \neq 0
\end{aligned}
$$

for each $(t, v) \in\left[0, \frac{1}{2}\right] \times[0,1]$, and

$$
\begin{aligned}
& p_{0}(t, v) \overline{p_{1}(t, v)}+\tilde{p}_{0}(t, v) \overline{\tilde{p}_{1}(t, v)}=0, \\
& \tilde{p}_{2}\left(t+\frac{1}{2}, v\right) \overline{\tilde{p}_{3}\left(t+\frac{1}{2}, v\right)}+e^{2 \pi i v} p_{1}(t, v) \overline{p_{0}(t, v)}=0
\end{aligned}
$$

for a.e. $(t, v) \in\left[0, \frac{1}{2}\right) \times[0,1)$. Put

$$
\begin{aligned}
& A=\min \left\{P_{l}(t, v): 0 \leq l \leq 4,(t, v) \in\left[0, \frac{1}{2}\right] \times[0,1]\right\}, \\
& B=\max \left\{P_{l}(t, v): 0 \leq l \leq 4,(t, v) \in\left[0, \frac{1}{2}\right] \times[0,1]\right\} .
\end{aligned}
$$

Then we have the following example:

Example 4.5. Let $\mathbf{a}$ and $\mathbf{b}$ be defined as in (4.25). Define $\mathbf{g}=\left(g_{1}, g_{2}\right)$ by

$$
\Phi_{\mathbf{g}}(t, v)=\mathcal{K}_{3}(t, v) \text { for a.e }(t, v) \in[0,1) \times[0,1),
$$

then $G(\mathbf{g}, \mathbf{a}, \mathbf{b})$ is a Riesz basis for $L^{2}(\mathbb{R})$ with Riesz bounds $A$ and $B$.

Proof. By the continuity of $q_{l}(v), \tilde{q}_{l^{\prime}}(v)\left(l=0,1,2, l^{\prime}=0,1,2,3\right)$ and (4.27)(4.31), we have $0<A \leq B<\infty$, where $A$ and $B$ are defined as in (4.32) and (4.33). It is easy to check that

$$
\left\langle\Phi_{\mathbf{g}}^{*}(t, v) \Phi_{\mathbf{g}}(t, v) x, x\right\rangle=P_{0}(t, v)\left|x_{1}\right|^{2}+P_{2}(t, v)\left|x_{2}\right|^{2}+P_{1}(t, v)\left|x_{3}\right|^{2}
$$

for $x \in \mathbb{C}^{3}$ and a.e. $(t, v) \in\left[0, \frac{1}{2}\right) \times[0,1)$, and

$$
\left\langle\Phi_{\mathbf{g}}^{*}(t, v) \Phi_{\mathbf{g}}(t, v) x, x\right\rangle=P_{3}\left(t-\frac{1}{2}, v\right)\left|x_{1}\right|^{2}+P_{4}\left(t-\frac{1}{2}, v\right)\left|x_{2}\right|^{2}+P_{2}\left(t-\frac{1}{2}, v\right)\left|x_{3}\right|^{2}
$$

for $x \in \mathbb{C}^{3}$ and a.e. $(t, v) \in\left[\frac{1}{2}, 1\right) \times[0,1)$. So

$$
A\|x\|^{2} \leq\left\langle\Phi_{\mathbf{g}}^{*}(t, v) \Phi_{\mathbf{g}}(t, v) x, x\right\rangle \leq B\|x\|^{2}
$$

for $x \in \mathbb{C}^{3}$ and a.e. $(t, v) \in[0,1) \times[0,1)$.

This implies that $G(\mathbf{g}, \mathbf{a}, \mathbf{b})$ is a Riesz basis for $L^{2}(\mathbb{R})$ with Riesz bounds $A$ and $B$ by Theorem 1.2.

Now we conclude this article with a concrete example of Riesz basis and its dual. 
Example 4.6. Let $\mathbf{a}$ and $\mathbf{b}$ be defined as in (4.25). In Example 4.5, take

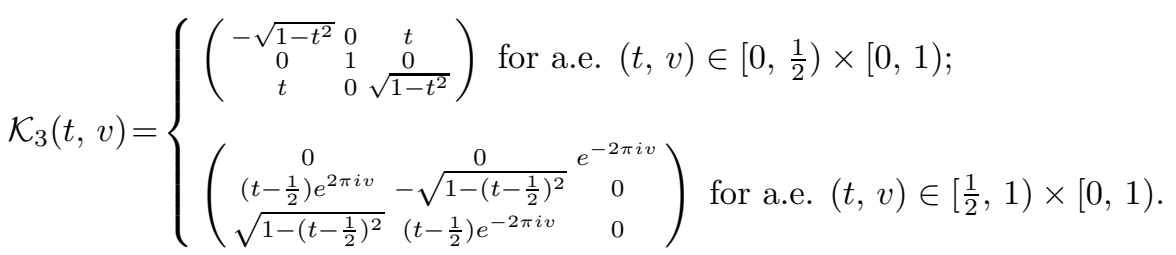

Define $\mathbf{g}=\left(g_{1}, g_{2}\right)$ by

$$
\Phi_{\mathbf{g}}(t, v)=\mathcal{K}_{3}(t, v) \text { for a.e. }(t, v) \in[0,1) \times[0,1) .
$$

Then

$$
\begin{aligned}
g_{1}(t)= & \chi_{\left[-\frac{1}{2}, 0\right)}(t)-\sqrt{1-t^{2}} \chi_{\left[0, \frac{1}{2}\right)}(t)+(t-2) \chi_{\left[2, \frac{5}{2}\right)}(t), \\
g_{2}(t)= & t \chi_{\left[0, \frac{1}{2}\right)}(t)+\sqrt{1-\left(t-\frac{1}{2}\right)^{2}} \chi_{\left[\frac{1}{2}, 1\right)}(t) \\
& +\sqrt{1-(t-2)^{2}} \chi_{\left[2, \frac{5}{2}\right)}(t)+\left(t+\frac{3}{2}\right) \chi_{\left[-\frac{3}{2},-1\right)}(t),
\end{aligned}
$$

and $G(\mathbf{g}, \mathbf{a}, \mathbf{b})$ is a Riesz basis for $L^{2}(\mathbb{R})$ by Example 4.5 . Thus $G(\mathbf{g}, \mathbf{a}, \mathbf{b})$ has a unique dual $G(\mathbf{h}, \mathbf{a}, \mathbf{b})$. By Theorem 1.3. From (4.35) we have

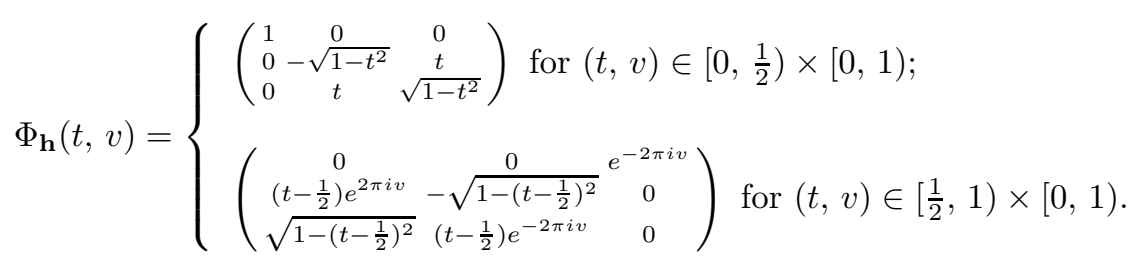

This implies that

$$
\begin{aligned}
h_{1}(t)= & \chi_{\left[0, \frac{1}{2}\right)}(t)+\left(t-\frac{1}{2}\right) \chi_{\left[\frac{1}{2}, 1\right)}(t)-\sqrt{1-\left(t+\frac{1}{2}\right)^{2}} \chi_{\left[-\frac{1}{2}, 0\right)}(t), \\
h_{2}(t)= & \left(t+\frac{3}{2}\right) \chi_{\left[-\frac{3}{2},-1\right)}(t)+\sqrt{1-\left(t-\frac{1}{2}\right)^{2}} \chi_{\left[\frac{1}{2}, 1\right)}(t) \\
& +(t-1) \chi_{\left[1, \frac{3}{2}\right)}(t)+\sqrt{1-(t-2)^{2}} \chi_{\left[2, \frac{5}{2}\right)}(t) .
\end{aligned}
$$

\section{References}

[1] M. A. Akinlar and J.-P. Gabardo, Oblique duals associated with rational subspace Gabor frames, J. Integral Equations Appl. 20 (2008), no. 3, 283-309.

[2] I. Daubechies, The wavelet transform, time-frequency localization and signal analysis, IEEE Trans. Inform. Theory 36 (1990), no. 5, 961-1005.

[3] H. G. Feichtinger and T. Strohmer, Gabor Analysis and Algorithms, Theory and Applications, Birkhäuser, Boston, 1998.

[4] _ Advances in Gabor Analysis, Birkhäuser, Boston, 2003.

[5] J.-P. Gabardo and D. Han, Balian-Low phenomenon for subspace Gabor frames, J. Math. Phys. 45 (2004), no. 8, 3362-3378. 
[6] - The uniqueness of the dual of Weyl-Heisenberg subspace frames, Appl. Comput. Harmon. Anal. 17 (2004), no. 2, 226-240.

[7] J.-P. Gabardo and Y.-Z. Li, Density results for Gabor systems associated with periodic subsets of the real line, J. Approx. Theory 157 (2009), no. 2, 172-192.

[8] K. Gröchenig, Foundations of Time-Frequency Analysis, Birkhäuser, Boston, 2001.

[9] C. Heil, History and evolution of the density theorem for Gabor frames, J. Fourier Anal. Appl. 13 (2007), no. 2, 113-166.

[10] F. Jaillet and B. Torrésani, Time-frequency jigsaw puzzle: adaptive multiwindow and multilayered Gabor expansions, Int. J. Wavelets Multiresolut. Inf. Process. 5 (2007), no. 2, 293-315.

[11] S. Jang, B. Jeong, and H. O. Kim, Compactly supported multiwindow dual Gabor frames of rational sampling density, Adv. Comput. Math. 38 (2013), no. 1, 159-186.

$[12]+$ Techniques for smoothing and splitting in the construction of tight frame Gabor windows, Int. J. Wavelets Multiresolut. Inf. Process. 11 (2013), no. 1, 1350005, 18pp.

[13] S. Li, Discrete multi-Gabor expansions, IEEE Trans. Inform. Theory 45 (1999), no. 6, 1954-1967.

[14] Y.-Z. Li and Q.-F. Lian, Gabor systems on discrete periodic sets, Sci. China Ser. A 52 (2009), no. 8, 1639-1660.

[15] Q.-F. Lian and Y.-Z. Li, The duals of Gabor frames on discrete periodic sets, J. Math. Phys. 50 (2009), no. 1, 013534, 22pp.

[16] Gabor frame sets for subspaces, Adv. Comput. Math. 34 (2011), no. 4, 391-411.

[17] A. Ron and Z. Shen, Affine systems in $L^{2}\left(\mathbb{R}^{d}\right)$ : the analysis of the analysis operator, J. Funct. Anal. 148 (1997), no. 2, 408-447.

[18] R. M. Young, An Introduction to Nonharmonic Fourier Series, Academic Press, New York, 1980

[19] Y. Y. Zeevi, Multiwindow Gabor-type representations and signal representation by partial information, Twentieth century harmonic analysis-a celebration (Il Ciocco, 2000), 173-199, NATO Sci. Ser. II Math. Phys. Chem., 33, Kluwer Acad. Publ., Dordrecht, 2001.

[20] Y. Y. Zeevi, M. Zibulski, and M. Porat, Multi-window Gabor schemes in signal and image representations, Gabor analysis and algorithms, 381-407, Appl. Numer. Harmon. Anal., Birkhäuser Boston, Boston, MA, 1998.

[21] M. Zibulski and Y. Y. Zeevi, Analysis of multiwindow Gabor-type schemes by frame methods, Appl. Comput. Harmon. Anal. 4 (1997), no. 2, 188-221.

YAN ZHANG

School of Mathematics and Information Science

Beifang University of Nationalities

Yinchuan 750021, P. R. China

E-mail address: yzhangbun@163.com

YUN-ZHANG LI

College of Applied Sciences

Beijing University of Technology

Beijing 100124, P. R. China

E-mail address: yzlee@bjut.edu.cn 